Algebraic 85 Geometric Topology

Volume 2 (2002) 51-93

Published: 5 February 2002

\title{
Formes différentielles généralisées sur une opérade et modèles algébriques des fibrations
}

\author{
DAvid Chataur
}

\begin{abstract}
We construct functors of generalized differential forms. In the case of nilpotent spaces of finite type, they determine the weak homotopy type of the spaces. Moreover they are equipped, in an elementary and natural way, with the action of cup-i products. Working with commutative algebras up to homotopy (viewed as algebras over a cofibrant resolution of the operad of commutative algebras), we show using these functors that the model of the fiber of a simplicial map is the cofiber of the algebraic model of this map.
\end{abstract}

Resumé On construit des foncteurs de formes différentielles généralisées. Ceux-ci, dans le cas d'espaces nilpotents de type fini, déterminent le type d'homotopie faible des espaces. Ils sont munis, d'une manière élémentaire et naturelle, de l'action de cup-i produits. Pour les algèbres commutatives à homotopit prés (algèbres sur une résolution cofibrante de l'opérade des algèbres commutatives), on démontre en utilisant les formes différentielles généralisées que le modèle de la fibre d'une application simpliciale est la cofibre du modèle de ce morphisme.

AMS Classification 18D50; 55P43, 55P48, 55T99

Keywords Modèles algébriques, formes différentielles, opérades, suites spectrales

\section{Introduction}

Il est bien connu que les cochaînes singulières d'un espace topologique forment une algèbre différentielle graduée. Celle-ci n'est pas commutative, mais commutative à homotopie près, ce défaut de commutativité se traduit par l'existence d'opérations sur la cohomologie à coefficients dans $\mathbb{F}_{p}$ : les opérations de Steenrod.

En outre, la théorie des opérades fournit un cadre agréable pour la construction de modèles algébriques pour les espaces topologiques. Une opérade est une 
structure algébrique permettant de coder un type d'algèbres (en particulier des types d'algèbres différentielles $\mathbb{Z}$-graduées). La théorie des algèbres sur une opérade semble tout adaptée à l'étude des structures algébriques à homotopie près. Pour les algèbres commutatives (algèbres sur l'opérade $\mathcal{C}$ om) on a la notion d'algèbres commutatives à homotopie près ou $E_{\infty}$-algèbres [26].

Aussi, V. Hinich et V. Schechtman ont montré que l'ont pouvait munir les cochaînes singulières d'un espace topologique, de manière naturelle, d'une structure de $E_{\infty}$-algèbre [21].

L'homotopie des $E_{\infty}$-algèbres et leur structure de catégorie modèle fermée ont été étudiées récemment par M. Mandell [31] et V. Hinich [19], [20]. V. Hinich a prouvé que la catégorie des opérades est une catégorie modèle fermée, et qu'une telle structure existe pour les algèbres sur une opérade cofibrante. On fixe un remplacement cofibrant $\mathcal{E}_{\infty}$ de l'opérade des algèbres commutatives; il est alors possible d'étudier l'homotopie des $\mathcal{E}_{\infty}$-algèbres, c'est-à dire l'homotopie des algèbres commutatives à homotopie près.

Pour une opérade $E_{\infty}$ particulière $\mathcal{C}$ (obtenue à partir de l'opérade des chaînes singulières de l'opérade des isométries linéaires), M. Mandell a montré une équivalence de catégories entre une sous-catégorie pleine de la catégorie homotopique des $\mathcal{C}$-algèbres sur $\overline{\mathbb{F}_{p}}$ et la catégorie homotopique des espaces topologiques nilpotents $\overline{\mathbb{F}_{p}}$-complets. V. Hinich a étendu ce résultat au cadre des $\mathcal{E}_{\infty}$-algèbres [20]. Enfin, M. Mandell a prouvé que deux espaces nilpotents de type finis sont faiblement homotopiquement équivalents si et seulement si leurs algèbres de cochaînes singulières sont $\mathcal{E}_{\infty}$-quasi-isomorphes [32].

Résultats Le cadre dans lequel on travaille est celui des opérades unitales augmentées dans la catégorie des $R$-modules différentiels $\mathbb{Z}$-gradués. On construit des foncteurs $\Omega^{\mathcal{O}}$ de formes différentielles généralisées pour les algèbres sur une opérade $\mathcal{O}$ (chapitre 3 ). Ces foncteurs sont l'analogue pour les $\mathcal{O}$ algèbres du foncteur $\mathcal{A}_{P l}$ de Sullivan pour les algèbres différentielles graduées commutatives. On montre que sous des hypothèses raisonnables (essentiellement que l'opérade choisie soit cofibrante) ces foncteurs permettent de calculer la cohomologie singulière à coefficients (théorème 3.1):

Théorème 1.1 Pour tout ensemble simplicial $X$ on a $\pi^{*} \Omega^{\mathcal{O}}(X) \cong H^{*}(X, R)$.

La preuve de ce résultat repose essentiellement sur des techniques de modèles acycliques adaptées au cadre des $R$-modules différentiels $\mathbb{Z}$-gradués. Toujours en utilisant la théorie des modèles acycliques et la structure de catégorie 
modèle fermée pour les opérades unitales augmentées, on montre que les foncteurs formes différentielles généralisées sont tous munis d'une structure de $\mathcal{E}_{\infty}$ algèbre. Ce résultat généralise ceux de $\mathrm{V}$. Hinich et $\mathrm{V}$. Schechtman obtenus pour les cochaînes singulières [21]. Si on travaille avec des $\mathcal{O}$-algèbres à coefficients dans $\mathbb{F}$ un corps de caractéristique positive, cette structure de $\mathcal{E}_{\infty}$-algèbre implique le résultat suivant (théorème 3.4 ):

Théorème 1.2 Pour tout ensemble simplicial $X$ l'algèbre $\Omega^{\mathcal{O}}(X)$ est munie de l'action d'opérations. Cette action induit sur $\pi^{*} \Omega^{\mathcal{O}}(X)$ une structure d'algèbre instable sur l'algèbre de Steenrod telle que $\pi^{*} \Omega^{\mathcal{O}}(X) \cong H^{*}(X, \mathbb{F})$ soit un isomorphisme d'algèbres instables.

Un des intérêts des formes différentielles généralisées est qu'elle permettent de travailler avec n'importe quel remplacement cofibrant de l'opérade $\mathcal{C}$ om. De plus la structure de $\mathcal{E}_{\infty}$-algèbre n'est pas très "lisible" sur les cochaînes singulières (les résultats de V. Hinich et V. Schechtman donnent seulement son existence, pour une description combinatoire de cette structure on pourra se référer aux travaux de $\mathrm{C}$. Berger et $\mathrm{B}$. Fresse [2]); alors que celle-ci est immédiate pour le foncteur des formes différentielles généralisées en $\mathcal{E}_{\infty}$-algèbres. Pour un choix judicieux d'une opérade $E_{\infty}$, on peut donner des représentants canoniques pour les cup-i produits.

Ces formes différentielles permettent de construire, de manière élémentaire, une paire de foncteurs adjoints (en fait une paire de foncteurs adjoints de Quillen) entre la catégorie des ensembles simpliciaux et de nombreuses catégories d'algèbres sur une opérade. Enfin, comme en théorie de Sullivan, le foncteur $\Omega^{\mathcal{O}}$ permet de définir un objet chemin naturel pour les $\mathcal{O}$-algèbres, et aussi des espaces fonctionnels simpliciaux pour ces mêmes $\mathcal{O}$-algèbres.

Grâce à l'introduction de formes différentielles généralisées à coefficients locaux et à l'extension de ces théories aux ensembles bisimpliciaux, on donne une construction de la suite spectrale de Leray-Serre (théorème 4.1):

Théorème 1.3 Soit $f: E \longrightarrow B$ une fibration entre ensembles simpliciaux de fibre $F$ (On suppose que la cohomologie de la base ou celle de la fibre est finie). Il existe une suite spectrale qui converge vers $H^{*}(E ; k)$ telle que

$$
E_{0}^{r, s}=\Omega^{\mathcal{O}, r}\left(B ; \mathcal{F}^{s}\right)
$$

pour un certain système de coefficients locaux $\mathcal{F}$. Quand celui-ci est simple (par exemple si $B$ est 1-connexe) le terme $E_{2}$ de cette suite spectrale s'écrit:

$$
E_{2}^{r, s}=H^{r}\left(B ; H^{s}(F ; k)\right) .
$$


Pour les $\mathcal{E}_{\infty}$-algèbres cette suite spectrale nous permet de construire un modèle algébrique de la fibre d'une application simpliciale cf théorème 4.2. Ainsi, on obtient un résultat qui est classique en homotopie rationnelle: Le modèle algébrique de la fibre correspond à la cofibre du modèle. Le théorème 4.2 s'inscrit dans la lignée des travaux de K. Hess et N. Dupont [7]. Il possède l'avantage de rester valide sur les entiers, mais aussi de donner un modèle de la fibre qui détermine son type d'homotopie. On espère aussi appliquer ce résultat à l'étude des espaces de lacets et des espaces de lacets libres. On retrouve aussi dans ce cadre algébrique les résultats de Kudo sur la transgression dans la suite spectrale de Leray-Serre.

Remerciements Ce travail est en grande partie issu de ma thèse "Formes différentielles sur une opérade et modèles algébriques pour les espaces topologiques". J'en profite donc pour remercier Jean-Louis Loday et Lionel Schwartz dont les conseils de rédaction m'ont été très utiles. Je tiens aussi à exprimer toute ma gratitude à Benoît Fresse pour ses nombreux conseils et l'attention bienveillante qu'il apporte à mes recherches. Enfin je remercie très chaleureusement mon directeur de thèse Marc Aubry pour sa disponibilité, son soutien infaillible et ses encouragements constants.

\section{Homotopie des opérades et des algèbres sur une opérade}

\section{$2.1 \quad$ Opérades}

La structure d'opérade a été utilisée et introduite au début des années 1970 par J. M. Boardman, R. M. Vogt [3] et J. P. May [34] dans un contexte topologique afin d'étudier l'homotopie des espaces de lacets itérés. V. Ginzburg, M. Kapranov [16] et E. Getzler, J.D.S. Jones [15] ont repris cette notion dans le cadre algébrique. On peut également se référer à l'exposé Bourbaki de J.L. Loday [28] ou à la première partie du livre de I. Kriz et J. P. May [26].

La catégorie des $R$-modules différentiels $\mathbb{Z}$-gradués On fixe un anneau $R$, que l'on suppose commutatif et unitaire. Les opérades avec lesquelles on travaille sont des opérades dans la catégorie monoïdale symétrique des $R$-modules différentiels $\mathbb{Z}$-gradués que l'on note $\mathbf{R} \mathcal{M}_{\mathbf{d g}}$. Par convention, la différentielle augmente le degré de 1.

Soit $M$ un objet de la catégorie $\mathbf{R} \mathcal{M}_{\mathbf{d g}}$. On note $M^{n}$ avec $n \in \mathbb{Z}$ le $R$-module des cochaînes de degré $n$. 
On forme un complexe

$$
\ldots \stackrel{d}{\longrightarrow} M^{n-1} \stackrel{d}{\longrightarrow} M^{n} \stackrel{d}{\longrightarrow} \ldots
$$

avec les composantes et la différentielle de notre $R$-module. Le $R$-module gradué $\pi^{*} M$ est la cohomologie de ce complexe.

Le produit tensoriel de deux $R$-modules différentiels $\mathbb{Z}$-gradués $M$ et $N$ s'écrit:

$$
(M \otimes N)^{n}=\bigoplus_{p+q=n} M^{p} \otimes N^{q} .
$$

La différentielle d'un tenseur étant donnée par la formule:

$$
d(m \otimes n)=d m \otimes n+(-1)^{|m|} m \otimes d n .
$$

On a un opérateur d'échange:

$$
\begin{gathered}
T: A \otimes B \longrightarrow B \otimes A \\
a \otimes b \mapsto(-1)^{|a||b|} b \otimes a .
\end{gathered}
$$

Si $A$ est un $R$-module différentiel gradué, alors $A[m], m \in \mathbb{Z}$, désigne la $m$ suspension de $A$, le $R$-module différentiel gradué tel que $A[m]^{*}=A^{m+*}$.

$\Sigma_{*}$-modules Un $\Sigma_{*}$-module ou suite symétrique est la donnée d'un ensemble $\{M(n)\}_{n \in \mathbb{N}}$ d'objets de $\mathbf{R} \mathcal{M}_{\mathbf{d g}}$. Pour tout entier $n$, le $R$-module $M_{n}$ est muni d'une action à droite du groupe symétrique $\Sigma_{n}$. On rearque que les $\Sigma_{*}$-modules forment une catégorie monoidale symétrique.

Opérades Une opérade $\mathcal{O}$ dans la catégorie $\mathbf{R} \mathcal{M}_{\mathbf{d g}}$ est la donnée d'un $\Sigma_{*}$ module $\{\mathcal{O}(n)\}_{n \in \mathbb{N}}$, d'un morphisme $\eta: R \longrightarrow \mathcal{O}(1)$ appelé morphisme unité et de produits de composition

$$
\gamma: \mathcal{O}(k) \otimes \mathcal{O}\left(j_{1}\right) \otimes \ldots \otimes \mathcal{O}\left(j_{k}\right) \longrightarrow \mathcal{O}(j)
$$

définis pour $1 \leq k$ et $\sum j_{s}=j$. Ces produits de composition sont, dans un certain sens, associatifs, unitaires et équivariants (cf [26], Part 1, section 1).

Opérades unitaires augmentées On travaille avec des opérades unitaires augmentées. On note $\mathbf{O} \mathbf{p}_{\text {ua }}$ la catégorie formée par de telles opérades.

Un objet de $\mathbf{O p}_{\text {ua }}$ est une opérade $\mathcal{O}$ avec un morphisme d'augmentation

$$
\epsilon: \mathcal{O} \longrightarrow \mathcal{C o m} \text {. }
$$

On rappelle que l'opérade $\mathcal{C}$ om est l'opérade des algèbres différentielles graduées commutatives. Elle est donnée par la formule $\operatorname{Com}(i)=R$. 
On demande aussi que $\epsilon$ induise un isomorphisme sur les composantes de degrés 0 et 1 :

$$
\begin{aligned}
\mathcal{O}(0) & =\operatorname{Com}(0)=R \\
\mathcal{O}(1) & =\operatorname{Com}(1)=R .
\end{aligned}
$$

Un morphisme de $\mathbf{O p}_{\mathbf{u a}}$ est un morphisme d'opérades $f: \mathcal{O} \longrightarrow \mathcal{O}^{\prime}$ tel que $f_{0}=f_{1}=I d$.

La catégorie $\mathbf{O p}_{\mathbf{u a}}$ possède un objet initial qui est aussi un objet final on note $\mathbb{I}$ cette opérade. Celle-ci est telle que $\mathbb{I}(0)=\mathbb{I}(1)=R$ et $\mathbb{I}(i)=0$ pour $i>1$. On vérifie aussi que cette catégorie est complète et cocomplète (on forme les limites et les colimites dans la catégorie des opérades au-dessus de l'opérade Com).

Algèbres sur une opérade On fixe $\mathcal{O}$ une opérade unitale augmentée. Une algèbre sur $\mathcal{O}$ (on dit aussi $\mathcal{O}$-algèbre) est un $R$-module différentiel $\mathbb{Z}$-gradué $A$ muni de produits d'évaluation:

$$
\theta_{j}: \mathcal{O}(j) \otimes A^{\otimes j} \longrightarrow A
$$

associatifs, unitaires et équivariants (pour l'action de $\Sigma_{j}$ ).

On note $\mathcal{O}-\mathcal{A} \lg _{\text {dg }}$ la catégorie des $\mathcal{O}$-algèbres.

Le foncteur oubli de $\mathcal{O}-\mathcal{A} \lg _{\mathbf{d g}}$ vers $\mathbf{R} \mathcal{M}_{\mathbf{d g}}$ admet un adjoint à gauche, le foncteur $\mathcal{O}$-algèbre libre que l'on note aussi $\mathcal{O}$. Pour tout $R$-module différentiel $\mathbb{Z}$-gradué $M$, on définit $\mathcal{O}(\mathrm{M})$ la $\mathcal{O}$-algèbre libre sur $\mathrm{M}$ par:

$$
\mathcal{O}(M)=\bigoplus_{p \geq 0} \mathcal{O}(p) \otimes_{R\left[\Sigma_{p}\right]} M^{\otimes p}=R \oplus M \bigoplus_{p \geq 2} \mathcal{O}(p) \otimes_{R\left[\Sigma_{p}\right]} M^{\otimes p} .
$$

Si $\mathcal{O}$ est une opérade dans la catégorie $\mathbf{R} \mathcal{M}_{\mathbf{d g}}$ alors $\pi^{*} \mathcal{O}$ est une opérade dans la catégorie des $\mathrm{R}$-modules $\mathbb{Z}$-gradués.

De plus, si $A$ est une $\mathcal{O}$-algèbre alors $\pi^{*} A$ est une $\pi^{*} \mathcal{O}$-algèbre.

\subsection{La théorie homotopique des opérades}

Les opérades sont un langage pour aborder l'étude d'espaces en topologie algébrique. Il parait naturel de se placer dans un cadre homotopique. Celui de Quillen ([9], [23], [36]) semble convenir tout à fait.

Homotopie des R-modules différentiels gradués D. Quillen a démontré que l'on pouvait munir la catégorie des $R$-modules différentiels $\mathbb{N}$-gradués d'une structure de catégorie modèle fermée [36]. Cette construction s'étend 
au cadre des $R$-modules différentiels $\mathbb{Z}$-gradués ([23],[39]). On a alors un structure de catégorie modèle pour laquelle les équivalences faibles sont les quasiisomorphismes et les fibrations sont les surjections. On remarque que tous les objets sont fibrants.

Sur les R-modules différentiels gradués cofibrants Pour tout $A$ les conditions suivantes sont équivalentes (cf [37]):

(a) Le complexe $A$ est cofibrant.

(b) Pour tout complexe acyclique $S$ le complexe $\operatorname{Hom}(A, S)$ est aussi acyclique.

En conséquence, on en déduit que si $A$ est un objet cofibrant, alors pour tout entier $A^{n}$ est un $R$-module projectif. Réciproquement tout complexe de $R$ modules projectifs borné supérieurement est cofibrant.

Homotopie des $\Sigma_{*}$-modules La catégorie des $\Sigma_{*}$-modules possède une structure de catégorie modèle fermée qui provient de la structure de catégorie modèle fermée des $R\left[\Sigma_{n}\right]$-modules différentiels gradués. Explicitement:

i) Un morphisme $f_{*}: M_{*} \longrightarrow N_{*}$ est une équivalence faible si pour tout $n \in \mathbb{N}$ l'application $f_{n}: M_{n} \longrightarrow N_{n}$ est un quasi-isomorphisme de $R\left[\Sigma_{n}\right]$-module différentiel gradué.

ii) Un morphisme $f_{*}: M_{*} \longrightarrow N_{*}$ est une fibration si pour tout $n \in \mathbb{N}$ le morphisme $f_{n}$ est un épimorphisme.

iii) Un morphisme $f_{*}: M_{*} \longrightarrow N_{*}$ est une cofibration si pour tout $n \in \mathbb{N}$ le morphisme $f_{n}$ est une cofibration.

\section{Homotopies des opérades}

Opérades unitaires augmentées libres On note $\Sigma_{*} \bmod ^{2}$ la catégorie des $\Sigma_{*}$-modules 2-réduits. Un objet $\mathcal{M}$ de $\Sigma_{*} \bmod ^{2}$ est un $\Sigma_{*}$-module tel que $\mathcal{M}_{0}=\mathcal{M}_{1}=0$.

Soit $\mathcal{R}$ le $\Sigma_{*}$-module 2-réduit tel que $\mathcal{R}_{n}=R$ pour tout entier $n \geq 2$. On introduit $\Sigma_{*} \mathbf{m o d}_{a}^{2}$ la catégorie des $\Sigma_{*}$-modules 2-réduits augmentés comme étant la catégorie des objets de $\Sigma_{*} \bmod ^{2}$ au-dessus de $\mathcal{R}$.

On remarque que la catégorie des $\Sigma_{*}$-modules 2-réduits augmentés est munie d'une structure de catégorie modèle fermée. En effet, $\Sigma_{*} \mathbf{m o d}^{2}$ est une catégorie modèle fermée. De plus on rappelle que si $X$ est un objet d'une catégorie modèle fermée $\mathcal{C}$, alors la catégorie des objets au-dessus de $X$ est aussi une catégorie modèle fermée. Les fibrations sont les épimorphismes et les équivalences faibles sont les quasi-isomorphismes. 
Soit $U: \mathbf{O p}_{u a} \longrightarrow \Sigma_{*} \mathbf{m o d}_{a}^{2}$ le foncteur oubli de la catégorie des opérades unitaires augmentées dans la catégorie des $\Sigma_{*}$-modules 2-réduits augmentés. Le foncteur $U$ admet un foncteur adjoint à gauche le foncteur opérade unitaire augmentée libre noté $\mathbb{T}_{u a}$ (Appendice B de [1]).

Une opérade $\mathcal{O}$ est dite quasi-libre s'il existe un $\Sigma_{*}$-module $M_{*}$ tel que $\mathbb{T}_{u a}\left(M_{*}\right)$ soit isomorphe à $\mathcal{O}$ en tant qu'opérade graduée.

V. Hinich montre que les opérades forment une catégorie modèle fermée (cf [19]). Il est possible d'adapter ce résultat au cadre unital augmenté.

Théorème 2.1 La catégorie des opérades unitaires augmentées est munie d'une structure de catégorie modèle fermée pour laquelle

i) Un morphisme $f: \mathcal{O} \longrightarrow \mathcal{P}$ est une équivalence faible si pour tout $n \in$ $\mathbb{N}$ l'application $f(n): \mathcal{O}(n) \longrightarrow \mathcal{P}(n)$ est un quasi-isomorphisme de $R\left[\Sigma_{n}\right]$ modules différentiels gradués.

ii) Un morphisme $f: \mathcal{O} \longrightarrow \mathcal{P}$ est une fibration si pour tout $n \in \mathbb{N}$ le morphisme $f(n)$ est un épimorphisme.

Proof On reprend les arguments de V. Hinich. On transporte la structure de catégorie modèle fermée des $\Sigma_{*}$-modules 2 -réduits vers les opérades unitaires augmentées via le foncteur $\mathbb{T}_{u a}$.

Il suffit de vérifier que si $\mathrm{M}$ est un $\Sigma_{*}$-module 2-réduit cofibrant acyclique $\left(\pi^{*} M(n)=0\right.$ pour $\left.n \geq 2\right)$ alors pour toute opérade unitaire augmentée $\mathcal{O}$ le morphisme canonique

$$
\mathcal{O} \longrightarrow \mathcal{O} \amalg \mathbb{T}_{u a}(M)
$$

est un quasi-isomorphisme.

Ce point se démontre facilement par extension d'une homotopie contractante de $M$ à l'opérade $\mathbb{T}_{u a}(M)$. Les formules d'extension de $\mathrm{V}$. Hinich restent valables dans notre cadre.

On a pour cette structure la caractérisation suivante des objets cofibrants:

Proposition 2.1 Une opérade est cofibrante si et seulement si celle-ci est une rétraction d'une opérade quasi-libre.

Proof La proposition repose sur le fait que la catégorie $\mathcal{O}_{u a}$ est engendrée de manière cofibrante. 
Homotopie des algèbres sur une opérade Si on travaille sur un corps $\mathbb{K}$ de caractéristique nulle, nous savons que pour toute opérade $\mathcal{O}$ la catégorie des $\mathcal{O}$ algèbres est munie d'une structure de catégorie modèle fermée. Les équivalences sont les quasi-isomorphismes, les épimorphismes sont les fibrations, on donnera plus loin une description des cofibrations. On appelle cette structure la structure modèle adjointe. Cette terminologie provient de l'adjonction de Quillen entre la catégorie des $\mathcal{O}$-algèbres et la catégorie des $\mathbb{K}$-espaces vectoriels différentiels gradués.

Si on travaille sur un anneau $R$ quelconque ce résultat n'est pas toujours vrai. Par exemple, sur $\mathbb{F}_{p}$, la catégorie des $\mathbb{Z}$-algèbres différentielles graduées commutatives ne peut être munie d'une structure modèle adjointe (il existe une autre structure de catégorie modèle fermée pour ces algèbres [39]). Par contre, si $\mathcal{O}$ est une opérade; la catégorie des $\mathcal{O}$-algèbres est munie d'une structure modèle adjointe si et seulement si pour toute $\mathcal{O}$-algèbre $A$ et tout $n \in \mathbb{Z}$ le morphisme canonique

$$
A \longrightarrow A \amalg \mathcal{O}\left(x_{n}, d x_{n}\right)
$$

est un quasi-isomorphisme.

On retrouve l'analogue du résultat de V. Hinich [19] pour les opérades unitaires augmentées cofibrantes. En effet, si $\mathcal{O}$ est une opérade cofibrante, alors la catégorie des $\mathcal{O}$-algèbres admet une structure modèle adjointe. Supposons que $\mathcal{O}$ et $\mathcal{O}^{\prime}$ soient deux modèles cofibrants de la même opérade $\mathbb{O}$; alors la catégorie des $\mathcal{O}$-algèbres et la catégorie des $\mathcal{O}^{\prime}$-algèbres sont équivalentes au sens de Quillen. La preuve repose sur une filtration du coproduit pour une opérade quasi-libre (cf [20]).

Extensions libres Dans cette section on caractérise les cofibrations pour les $\mathcal{O}$-algèbres.

Notons $A \amalg B$ le coproduit de deux $\mathcal{O}$-algèbres (pour une réalisation de ce coproduit consulter [14]). On peut généraliser la notion d'extension libre (on parle aussi de morphisme quasi-libre) introduite dans le cadre des algèbres différentielles graduées [12] aux $\mathcal{O}$-algèbres.

Définition 2.1 Une extension libre est un morphisme de $\mathcal{O}$-algèbres

$$
A \stackrel{i}{\longrightarrow} A \amalg_{\tau} \mathcal{O}(M)
$$

tel que:

a) $A \amalg_{\tau} \mathcal{O}(M)=A \amalg \mathcal{O}(M)$ en tant que module gradué.

b) Le morphisme $i$ est l'application canonique. 
c) Le $R$-module $M$ s'écrit sous la forme $M=\bigcup_{i=0}^{\infty} M(i)$ avec $M(i) \subset M(i+1)$. Les $R$-modules gradués $M(0)$ et $M(i+1) / M(i)$ sont libres. d) La différentielle $d$ est telle que $d: M(0) \longrightarrow A$ et $d: M(i+1) \longrightarrow$ $A \amalg \mathcal{O}(M(i))$.

Toute application $f: A \longrightarrow B$ admet une factorisation:

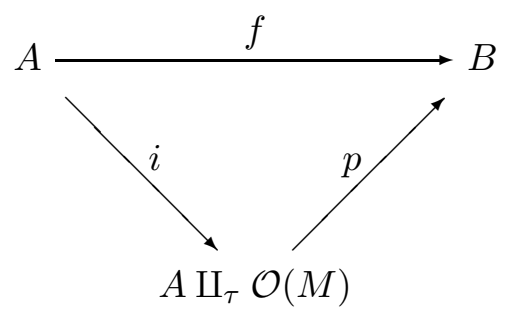

avec $i$ une extension libre et $p$ une fibration acyclique.

De plus, un morphisme est une cofibration si et seulement si c'est une rétraction d'une extension libre. Ceci repose sur le fait que la catégorie des $\mathcal{O}$-algèbres est engendrée de manière cofibrante. On peut montrer que la catégorie des $\mathcal{O}$ algèbres est une catégorie modèle cellulaire [22], [23] (ce qui est aussi le cas de la catégorie des opérades unitaires augmentées et de la catégorie des $\Sigma_{*}$-modules).

\subsection{Sur les $\mathcal{O}$-algèbres à homotopie près}

Soit $\mathbb{( O}$ une opérade unitaire et augmentée, on fixe un modèle cofibrant $\mathcal{O}$ de cette opérade.

Définition 2.2 On appelle $\mathbb{O}$-algèbre à homotopie près un objet de la catégorie des $\mathcal{O}$-algèbres.

Nous avons vu précédemment que du point vue homotopique le choix du modèle cofibrant importait peu (pour deux modèles cofibrants de la même opérade les catégories homotopiques sont équivalentes).

Une des difficultés consiste à construire un modèle cofibrant explicite pour une opérade $\mathbb{0}$ donnée.

Dans le cadre rationnel et pour les opérades de Koszul ([16], [28]), il existe un procédé utilisant la construction cobar $B^{*}$ pour obtenir un tel modèle. Si $\mathbb{O}$ est une opérade de Koszul, si $\mathbb{O}^{!}$est son dual de Koszul, alors $B^{*}\left(\mathbb{O}^{!}[-1]\right)^{*}$ est 
une résolution quasi-libre de $\mathbb{O}$. Toujours dans le cadre rationnel une théorie du modèle minimal a été développé par M. Markl [33].

Toute $\mathbb{O}$-algèbre est une $\mathcal{O}$-algèbre via le morphisme d'opérades $\mathcal{O} \longrightarrow \mathbb{O}$.

On s'intéresse plus spécifiquement au cas des algèbres commutatives à homotopie près. On choisit un modèle cofibrant de l'opérade $\mathcal{C}$ om que l'on note $\mathcal{E}_{\infty}$. On dit que $\mathcal{E}_{\infty}$ est une $E_{\infty}$-opérade cofibrante. On peut même supposer que l'opérade $\mathcal{E}_{\infty}$ est quasi-libre. Dans ce cas, les modules différentiels gradués $\mathcal{E}_{\infty}(n)$ sont tous $R\left[\Sigma_{n}\right]$-libres et acycliques. On peut choisir ces modules différentiels $\mathbb{Z}$-gradués tels que $\mathcal{E}_{\infty}(n)^{p}=0$ pour $p>0$.

Dans le langage de P. May [26] une $E_{\infty}$-opérade $\mathcal{O}$ est une opérade telle que $\mathcal{O}(l)$ soit une résolution $R\left[\Sigma_{l}\right]$-projective de $R$. On remarque qu'une $E_{\infty}$-opérade au sens de P. May n'est pas nécessairement cofibrante en tant qu'opérade.

Si on travaille avec une $E_{\infty}$-opérade $\mathcal{O}$ et avec $R$ un corps de caractéristique positive, alors l'homotopie des $\mathcal{O}$-algèbres est munie d'opérations:

Soient $\mathcal{O}$ une $E_{\infty}$-opérade et $A$ une $\mathcal{O}$-algèbre, il existe pour tout $s \geq 0$ et $R$ de caractéristique 2 des opérations:

$$
\mathcal{P}^{s}: \pi^{q} A \longrightarrow \pi^{q+s} A
$$

et pour $R$ de caractéristique $p>2$ :

$$
\mathcal{P}^{s}: \pi^{q} A \longrightarrow \pi^{q+2 s(p-1)} A .
$$

Ces opérations vérifient les propriétés suivantes:

i) $\mathcal{P}^{s}(x)=0$ si $p=2$ et $s<|x|$ ou si $p>2$ et $2 s<|x|$.

ii) $\mathcal{P}^{s}(x)=x^{p}$ si $p=2$ et $s=|x|$ ou si $p>2$ et $2 s=|x|$.

iii) $\mathcal{P}^{s}(x y)=\sum \mathcal{P}^{t}(x) \mathcal{P}^{s-t}(y)$ (formule de Cartan).

iv) (formule d'Adem) Si $p \geq 2$ et $t>p s$ :

$$
\mathcal{P}^{t} \mathcal{P}^{s}=\sum_{i}(-1)^{t+i}(p i-t, t-(p-1) s-i) \mathcal{P}^{s+t-i-1} \mathcal{P}^{i}
$$

si $p>2, t>p s$, et si par $\beta$ on note le mod- $p$ Bockstein, alors:

$$
\begin{gathered}
\mathcal{P}^{t} \beta \mathcal{P}^{s}=\sum_{i}(-1)^{t+i}(p i-t, t-(p-1) s-i) \beta \mathcal{P}^{s+t-i-1} \mathcal{P}^{i} \\
-\sum_{i}(-1)^{t+i}(p i-t-1, t-(p-1) s-i) \mathcal{P}^{s+t-i-1} \beta \mathcal{P}^{i}
\end{gathered}
$$

$(i, j)=\frac{(i+j) !}{i ! j !}$ si $i \geq 0$ et $j \geq 0$ et $(i, j)=0$ si $i$ ou $j$ sont négatifs. 
Exemple de $E_{\infty}$-opérade: la résolution bar des groupes symétriques Soit $\mathcal{R}_{B}$ le $\Sigma_{*}$-module tel que $\mathcal{R}_{B}(n)$ est le complexe normalisé de la résolution bar du groupe symétrique $\Sigma_{n}$. Ce $\Sigma_{*}$-module est une opérade. L'opérade $\mathcal{R}_{B}$ est évidemment une $E_{\infty}$-opérade au sens de P. May, mais elle n'est pas cofibrante (l'opérade $\mathcal{C}$ om est rétracte de cette opérade). Les cogèbres sur cette opérade ont été étudiées par J. Smith [40]. V.A. Smirnov a lui ausi étudié les cogèbres sur une $E_{\infty}$-opérade et leurs liens avec l'homotopie des espaces topologiques [41], [42].

\section{Formes différentielles généralisées}

Dans ce chapitre on construit des foncteurs de formes différentielles généralisées. Ce sont des foncteurs de la catégorie des ensembles simpliciaux vers une catégorie de $\mathcal{O}$-algèbres.

Grâce à la théorie de modèles acycliques que nous développons dans le premier paragraphe on établit une équivalence d'homotopie entre ces foncteurs et le foncteur des cochaînes singulières normalisées (c'est le résultat principal du second paragraphe).

Dans le troisième paragraphe, on étudie la structure multiplicative de ces foncteurs et on montre qu'ils sont tous à valeurs dans les $\mathcal{E}_{\infty}$-algèbres.

Enfin dans le dernier paragraphe, on construit une paire de foncteurs adjoints de Quillen entre les ensembles simpliciaux et les $\mathcal{O}$-algèbres, via un foncteur de formes différentielles généralisées et un foncteur de réalisation simpliciale. On donne aussi quelques applications à l'homotopie des $\mathcal{O}$-algèbres (espace de chemins et homotopie simpliciale).

\subsection{La théorie des modèles acycliques}

On étend la théorie des modèles acycliques [11], [30], [38] au cadre $\mathbb{Z}$-gradué.

Soit $F: \mathcal{S}^{o p} \longrightarrow \mathbf{C}$ un foncteur contravariant des ensembles simpliciaux à valeurs dans une catégorie $\mathbf{C}$. On associe à $F$ le foncteur contravariant $F^{\prime}$ : $\mathcal{S}^{o p} \longrightarrow \mathbf{C}$ tel que:

$$
F^{\prime}(X)=\prod_{x \in X_{n}} F(\Delta[n])
$$

où $\Delta[n]$ est le simplexe standard de dimension $n$. Le produit est pris sur tous les $n \geq 0$ et les $x \in X_{n}$. On notera $\left\{m_{x}, x\right\} \in F^{\prime}(X)$ l'élément dont 
la composante indexée par $x \in X_{n}$ est l'élément $m_{x} \in F^{\prime}(\Delta[n])$. Soit $f$ : $X \longrightarrow Y$ un morphisme entre ensembles simpliciaux. Le morphisme associé $F^{\prime}(f): F^{\prime}(Y) \longrightarrow F^{\prime}(X)$ est donné par

$$
F^{\prime}(f)\left\{m_{y}, y\right\}=\left\{m_{f(x), x}\right\}_{y=f(x)} .
$$

Une transformation naturelle $T: F \longrightarrow G$ induit $T^{\prime}: F^{\prime} \longrightarrow G^{\prime}$ donnée par la formule:

$$
\left.T^{\prime}(X)\left\{m_{x}, x\right\}=\left\{T(\Delta[n]) m_{x}, x\right)\right\} .
$$

On définit aussi une transformation naturelle $\Phi: F \longrightarrow F^{\prime}$ en posant

$$
\Phi(X) u=\{F(\mathbf{x}) u, x\}
$$

pour $u \in F(X)$. Dans cette formule, on utilise le fait que la donnée de $x \in X_{n}$ est équivalente à un morphisme $\mathbf{x}: \Delta[\mathbf{n}] \longrightarrow \mathbf{X}$. On vérifie facilement la formule $T^{\prime} \Phi=\Phi T$.

Définition 3.1 a) On dit que $F: \mathcal{S}^{o p} \longrightarrow \mathbf{R} \mathcal{M}_{\mathbf{d g}}$ est coreprésentable s'il existe une transformation naturelle $\Psi: F^{\prime} \longrightarrow F$ telle que $\Psi$ est inverse à gauche de $\Phi$.

b) Un foncteur $F: \mathcal{S}^{o p} \longrightarrow \mathbf{R} \mathcal{M}_{\mathbf{d g}}$ est augmenté s'il existe une transformation naturelle $\epsilon: F \longrightarrow R$. On suppose que pour tout $n$ le morphisme $\epsilon: F(\Delta[n]) \longrightarrow R$ est une fibration.

c) On dit que $F$ est acyclique sur les modèles, si pour tout $n \in \mathbb{N}$, l'augmentation $\epsilon: F(\Delta[n]) \longrightarrow R$ est une équivalence faible.

d) Le foncteur $F$ est cofibrant, si pour tout $n \in \mathbb{N}, F(\Delta[n])$ est cofibrant.

Proposition 3.1 Supposons que $F: \mathcal{S}^{o p} \longrightarrow \mathbf{C}$ est un foncteur coreprésentable, augmenté, acyclique sur les modèles et que le foncteur $G: \mathcal{S}^{\text {op }} \longrightarrow \mathbf{C}$ est augmenté, cofibrant.

Alors, il existe une transformation naturelle $f: G \longrightarrow F$ telle que $\epsilon f=\epsilon$.

De plus, deux transformations naturelles $f, g: G \longrightarrow F$ telles que $\epsilon f=\epsilon=\epsilon g$, sont homotopes. Plus précisément, il existe une homotopie à gauche (naturelle) $h: G \longrightarrow F$ entre $f$ et $g$.

Preuve i) Considérons le diagramme suivant:

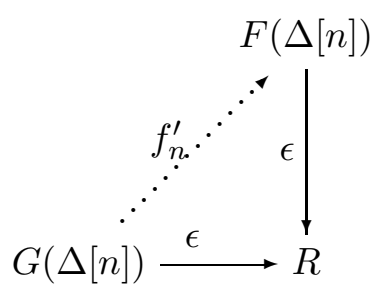

Algebraic $\& 3$ Geometric Topology, Volume 2 (2002) 
l'application verticale est une fibration acyclique (par hypothèse), et $G(\Delta[n])$ est cofibrant. Sous ces hypothèses, il existe un relèvement $f_{n}^{\prime}$. On obtient ainsi une transformation naturelle $f^{\prime}: F^{\prime} \longrightarrow G^{\prime}$. On pose $f=\Psi f^{\prime} \Phi$, c'est la transformation naturelle demandée.

ii) Comme le relèvement est unique à une homotopie à gauche près, on en déduit une famille d'homotopie à gauche $H_{n}^{\prime}$ de $f_{n}^{\prime}$ à $g_{n}^{\prime}$.

Comme il existe dans $\mathbf{R} \mathcal{M}_{\mathbf{d g}}$ un objet cylindre naturel qui commute avec les produits, ceci permet de construire une homotopie $H^{\prime}: I F^{\prime} \longrightarrow G^{\prime}$ de $f$ à $g$. Grâce à la naturalité de ce même objet chemin on a une homotopie à gauche $H: I F \longrightarrow G$.

Corollaire 3.1 Si $F$ et $G$ sont tous deux coreprésentables, augmentés, cofibrants et acycliques sur les modèles, alors $F$ et $G$ sont naturellement homotopiquement équivalents.

Toute transformation naturelle entre deux foncteurs coreprésentables, augmentés, cofibrants et acycliques sur les modèles qui commute aux augmentations induit une équivalence d'homotopie.

On note $\mathcal{C}: \mathcal{S}^{o p} \longrightarrow \mathbf{R} \mathcal{M}_{\mathbf{d g}}$ le foncteur des cochaînes singulières normalisées. Le foncteur $\mathcal{C}$ est coreprésentable, augmenté, cofibrant et acyclique sur les modèles. On en déduit le résultat suivant:

Proposition 3.2 Soit $F: \mathcal{S}^{o p} \longrightarrow \mathbf{R} \mathcal{M}_{\mathrm{dg}}$ un foncteur contravariant qui est coreprésentable, augmenté, cofibrant et acyclique sur les modèles, et $\mathcal{C}: \mathcal{S}^{o p}$ $\mathbf{R} \mathcal{M}_{\mathbf{d g}}$ le foncteur des cochaînes singulières normalisées.

Alors, les foncteurs $F$ et $\mathcal{C}$ sont naturellement équivalents. Donc, pour tout ensemble simplicial $X$ on a un isomorphisme naturel:

$$
\pi^{*} F(X) \cong \pi^{*} C(X) \cong H^{*}(X ; R) .
$$

\subsection{Le foncteur des formes différentielles généralisées pour les algèbres sur une opérade}

Nous construisons dans ce qui suit un foncteur de formes différentielles généralisées pour les algèbres sur une opérade $\mathcal{O}$. On prouve que ce foncteur qui est noté $\Omega^{\mathcal{O}}$ vérifie les hypothèses de la proposition ci-dessus. Pour être plus précis, on fixe $\mathcal{O}$ une opérade unitaire augmentée avec les propriétés suivantes:

a) Le morphisme d'augmentation $\epsilon: \mathcal{O} \longrightarrow \mathcal{C}$ om est une fibration. 
b) Le $R$-module différentiel $\mathcal{O}(n)$ est concentré en degrés négatifs.

c) L'opérade $\mathcal{O}$ est cofibrante.

L'hypothèse a) permet de définir une suite d'applications $s_{n}: R \longrightarrow \mathcal{O}(n)$ telles que $\epsilon s_{n}=I d_{R}$. Les $\left\{s_{n}\right\}_{n \in \mathbb{N}}$ ne donnent en aucun cas un morphisme d'opérades, sinon l'opérade $\mathcal{C}$ om serait une rétraction de $\mathcal{O}$ et donc cofibrante.

Posons $1_{n}=s_{n}(1)$. Comme $\mathcal{O}(0)=\mathcal{O}(1)=R$ (car $\mathcal{O}$ est unitaire augmentée), on a $1_{0}=1_{1}=1$.

Proposition 3.3 Soit $A$ une $\mathcal{O}$-algèbre. Le morphisme structural

$$
\theta_{2}: \mathcal{O}(2) \otimes A^{\otimes 2} \longrightarrow A
$$

vérifie l'équation:

$$
\theta_{2}\left(1_{2} \otimes \lambda \otimes a\right)=\theta_{2}\left(1_{2} \otimes a \otimes \lambda\right)=\lambda . a
$$

pour tout $\lambda \in R$ et tout $a \in A$.

Proof On considère le produit $\gamma_{2}: \mathcal{O}(2) \otimes \mathcal{O}(0) \otimes \mathcal{O}(1) \longrightarrow \mathcal{O}(1)$. Le diagramme commutatif:

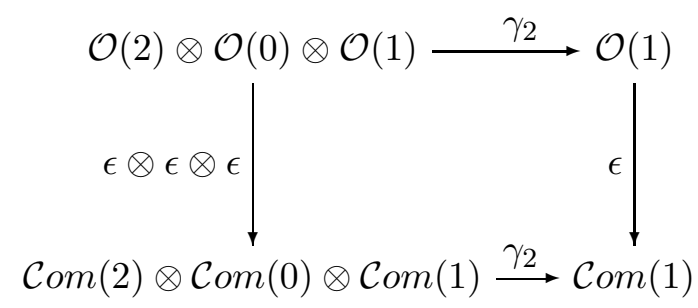

montre que $\gamma_{2}\left(1_{2} \otimes 1_{0} \otimes 1_{1}\right)=1_{1}=1$. Les relations de la proposition sont une conséquence de ces identités.

Cette proposition montre que pour toute $\mathcal{O}$-algèbre $A$ le produit:

$$
\begin{gathered}
\mu: A \otimes A \longrightarrow A \\
\mu(a \otimes b)=\theta_{2}\left(1_{2} \otimes a \otimes b\right)
\end{gathered}
$$

possède une unité. Cette propriété d'unitalité n'est pas vérifiée pour une $E_{\infty}$ opérade quelconque.

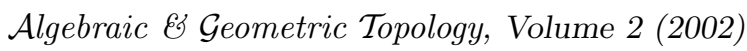


Définition 3.2 On a une $\mathcal{O}$-algèbre simpliciale $\Omega^{\mathcal{O}}{ }_{*}$. dont la composante de dimension simpliciale $n$ est la $\mathcal{O}$-algèbre

$$
\Omega^{\mathcal{O}}{ }_{n}=\frac{\mathcal{O}\left(x_{0}, \ldots, x_{n} ; d x_{0}, \ldots, d x_{n}\right)}{I_{n}}
$$

engendrée par les éléments $x_{0}, \ldots, x_{n}$ de degré 0 et $d x_{0}, \ldots, d x_{n}$ de degré 1 et quotientée par l'idéal $I_{n}$ engendré par les relations $\sum x_{i}=1_{0}, \sum d x_{i}=0$. Les opérateurs de face sont donnés par les formules:

$\delta_{j}\left(x_{j}\right)=0$ et $\delta_{j}\left(d x_{j}\right)=0 ;$

$\delta_{j}\left(x_{i}\right)=x_{i}$ et $\delta_{j}\left(d x_{i}\right)=d x_{i}$ si $i<j ;$

$\delta_{j}\left(x_{i}\right)=x_{i-1}$ et $\delta_{j}\left(d x_{i}\right)=d x_{i-1}$ si $i>j$;

les opérateurs de dégénérescence par les formules:

$\sigma_{j}\left(x_{j}\right)=x_{j}+x_{j+1}$ et $\sigma_{j}\left(d x_{j}\right)=d x_{j}+d x_{j+1}$;

$\sigma_{j}\left(x_{i}\right)=x_{i}$ et $\sigma_{j}\left(d x_{i}\right)=d x_{i}$ si $i<j$;

$\sigma_{j}\left(x_{i}\right)=x_{i+1}$ et $\sigma_{j}\left(d x_{i}\right)=d x_{i+1}$ si $i>j$.

On définit le foncteur des formes différentielles généralisées:

$$
\Omega^{\mathcal{O}}: \mathcal{S}^{o p} \longrightarrow \mathcal{O}-A l g_{d g}
$$

par la formule:

$$
\Omega^{\mathcal{O}}(X)=\operatorname{Hom}_{\mathcal{S}}\left(X, \Omega^{\mathcal{O}}{ }_{*}\right)
$$

On remarque que $\Omega^{\mathcal{O}}(\Delta[n])=\operatorname{Hom}_{\mathcal{S}}\left(\Delta[n], \Omega^{\mathcal{O}}{ }\right) \cong \Omega^{\mathcal{O}}{ }_{n}$. On va montrer que le foncteur des formes différentielles généralisées est coreprésentable, cofibrant et acyclique sur les modèles.

Si on applique la théorie des modèles acycliques, on déduit que ce foncteur est homotopiquement équivalent au foncteur des cochaînes singulières.

Lemme 3.1 Si l'opérade $\mathcal{O}$ est cofibrante et si chaque $R$-module différentiel $\mathbb{Z}$-gradué $\mathcal{O}(l)$ est concentré en degrés négatifs, alors $\Omega^{\mathcal{O}}{ }_{n}$ est cofibrant et acyclique relativement à l'augmentation.

Preuve Montrons d'abord que $\Omega^{\mathcal{O}}{ }_{n}$ est acyclique; à cette fin on remarque que l'on a l'isomorphisme de $\mathcal{O}$-algèbres:

$$
\frac{\mathcal{O}\left(x_{0}, \ldots, x_{n} ; d x_{0}, \ldots, d x_{n}\right)}{I_{n}} \cong \mathcal{O}\left(x_{1}, \ldots, x_{n} ; d x_{1}, \ldots, d x_{n}\right)
$$


Or $\mathcal{O}\left(x_{1}, \ldots, x_{n} ; d x_{1}, \ldots, d x_{n}\right)$ est acyclique. En effet, pour une opérade cofibrante une algèbre libre sur un R-module différentiel gradué acyclique est acyclique.

Il reste à montrer que $\Omega^{\mathcal{O}}$ est cofibrant en tant que $R$-module différentiel $\mathbb{Z}$-gradué.

On rappelle qu'un $\mathrm{R}$-module différentiel $\mathbb{Z}$-gradué projectif n'est pas nécessairement cofibrant [37]. Mais c'est le cas s'il est borné supérieurement.

En utilisant l'isomorphisme précédent $\Omega^{\mathcal{O}}{ }_{n}$, on a:

$$
\Omega^{\mathcal{O}}{ }_{n}=\bigoplus_{l \geq 0} \mathcal{O}(l) \otimes_{R\left[\Sigma_{l}\right]} M^{\otimes l}
$$

On pose $\Omega_{n}^{\mathcal{O}}(l)=\mathcal{O}(l) \otimes_{R\left[\Sigma_{l}\right]} M^{\otimes l}$. Montrons que ce R-module différentiel $\mathbb{Z}$-gradué est cofibrant, on en déduit alors que $\Omega^{\mathcal{O}}{ }_{n}$ est aussi cofibrant.

Comme $\mathcal{O}$ est cofibrante il existe une opérade quasi-libre $\mathcal{O}$ " telle que $\mathcal{O}$ est une rétraction de $\mathcal{O}$ ". Donc pour tout $l, \mathcal{O}(l)$ est un rétract de $\mathcal{O}$ " $(l)$. Et $\mathcal{O}$ " $(l)$ est un $R\left[\Sigma_{l}\right]$-module libre, car $\mathcal{O}$ " est une opérade libre et cofibrante. On peut supposer que cette opérade est aussi bornée supérieurement. Alors $\Omega^{\mathcal{O}}{ }_{n}(l)$ est un rétract de $\mathcal{O}^{\prime \prime}(l) \otimes_{R\left[\Sigma_{l}\right]} M^{\otimes l}$. L'objet $M$ est un R-module différentiel $\mathbb{Z}$-gradué libre concentré en degrés 0 et 1 . On en déduit facilement que $\mathcal{O}^{\prime \prime}(l) \otimes_{R\left[\Sigma_{l}\right]} M^{\otimes l}$ est cofibrant (car celui-ci est $R$-libre en tout degré et borné supérieurement).

Le résultat est une conséquence immédiate du fait que les objets cofibrants sont stables par rétraction.

Afin de montrer que le foncteur des formes différentielles généralisées est coreprésentable, il suffit de prouver que l'algèbre simpliciale $\Omega^{\mathcal{O}}$ * est contractile en tant qu'ensemble simplicial. En effet d'après M. Majewski [30], si $\mathbb{A}: \mathcal{S} \longrightarrow$ $\mathbf{R} \mathcal{M}_{\mathbf{d g}}$ est un foncteur de la catégorie des ensembles simpliciaux à valeurs dans $\mathbf{R} \mathcal{M}_{\mathbf{d g}}$ tel que $\mathbb{A}(X)=\operatorname{Hom}_{\mathcal{S}}\left(X, M^{*}\right)$, alors $\mathbb{A}$ est coreprésentable si $M^{*}$ est contractile.

Lemme 3.2 Pour tout $s \geq 1$, les groupes d'homotopie $\pi_{s-1}\left(\Omega^{\mathcal{O}}{ }_{*}\right)$ sont triviaux.

Preuve On étend à l'algèbre simpliciale $\Omega^{\mathcal{O}}$ * la preuve donnée par M. Karoubi dans le cadre des formes différentielles non commutatives ([24],[25]). On commence par montrer que $\pi_{0}\left(\Omega^{\mathcal{O}}{ }\right)$ est trivial. Soit $\omega \in \Omega^{\mathcal{O}}{ }_{0}$. On identifie $\Omega^{\mathcal{O}}{ }_{0}$ avec $R$, on suppose que $\omega$ est un scalaire. On identifie aussi $\Omega^{\mathcal{O}}{ }_{1}$ avec $\mathcal{O}(x, d x)$. 
L'application $\delta_{0}$ correspond à l'évaluation en $x=0, d x=0$ et $\delta_{1}$ à l'évaluation $x=1$ et $d x=0$. L'élément $\theta=\omega(1-x)$ vérifie $\delta_{0} \theta=\omega, \delta_{1} \theta=\omega$. D'où le résultat. On suppose $s \geq 2$. Montrer que $\pi_{s-1}\left(\Omega^{\mathcal{O}} *\right)$ est trivial est équivalent à prouver que, pour toute forme $\omega \in \Omega^{\mathcal{O}}{ }_{s-1}$ satisfaisant $\delta_{i} \omega=0$ pour tout $i$, il existe $\theta \in \Omega^{\mathcal{O}}$ s tel que $\delta_{0} \theta=\omega$ et $\delta_{i} \theta=0$ si $i>0$. Comme on a $1-t_{1}-\ldots-t_{s}=0$ et $d t_{1}+\ldots+d t_{s}=0$ dans $\Omega^{\mathcal{O}}{ }_{s-1}$ on remplace $t_{1}$ par $1-t_{2}-\ldots-t_{s}$ et $d t_{1}$ par $-d t_{2}-\ldots-d t_{s}$. Puis on pose

$$
\omega=\mu_{1}\left(t_{2}, \ldots, t_{s} ; d t_{2}, \ldots, d t_{s}\right)
$$

avec $\mu_{1} \in \mathcal{O}\left(t_{2}, \ldots, t_{s} ; d t_{2}, \ldots, d t_{s}\right)$. Enfin on définit $\sigma \in \Omega^{\mathcal{O}}{ }_{s}$ :

$$
\sigma_{1}\left(t_{0}, t_{1}, \ldots, t_{s} ; d t_{0}, \ldots, d t_{s}\right)=\gamma_{2}\left(1_{2} \otimes t_{1} \otimes \mu_{1}\left(t_{2}, \ldots, t_{s} ; d t_{2}, \ldots, d t_{s}\right)\right)
$$

On vérifie que les restrictions aux faces sont nulles pour $i>0$ et que la restriction à la 0 -face est de la forme $\theta_{2}\left(1_{2} \otimes t_{1} \otimes \omega\left(t_{1}, \ldots, t_{s} ; d t_{1}, \ldots, d t_{s}\right)\right)$. De manière analogue on construit pour chaque $i$ des formes $\sigma_{i}$ telles que:

$\delta_{j}\left(\sigma_{i}\right)=0$ pour $j \neq 0$,

$\delta_{0}\left(\sigma_{j}\right)=\theta_{2}\left(1_{2} \otimes t_{j} \otimes \omega\left(t_{1}, \ldots, t_{s} ; d t_{1}, \ldots, d t_{s}\right)\right)$.

Et la forme $\theta$ est donnée par la formule suivante:

$$
\theta=\sum_{1 \leq i \leq s} \sigma_{i}\left(t_{0}, t_{1}, \ldots, t_{s} ; d t_{0}, \ldots, d t_{s}\right) .
$$

Cette forme vérifie:

$\delta_{j}(\theta)=0$ pour $j \neq 0$,

$$
\begin{aligned}
\delta_{0}(\theta) & =\sum_{1 \leq i \leq s} \gamma_{2}\left(1_{2} \otimes t_{j} \otimes \omega\left(t_{1}, \ldots, t_{s} ; d t_{1}, \ldots, d t_{s}\right)\right) \\
\delta_{0}(\theta) & =\theta_{2}\left(1_{2} \otimes \sum_{1 \leq i \leq s} t_{j} \otimes \omega\left(t_{1}, \ldots, t_{s} ; d t_{1}, \ldots, d t_{s}\right)\right) \\
\delta_{0} & =\theta_{2}\left(1_{2} \otimes 1_{0} \otimes \omega\left(t_{1}, \ldots, t_{s} ; d t_{1}, \ldots, d t_{s}\right)\right) .
\end{aligned}
$$

Comme $\theta_{2}\left(1_{2} \otimes 1_{0} \otimes w\right)=w$ (d'après la proposition 3.3) on a:

$\delta_{0}(\theta)=\omega\left(t_{1}, \ldots, t_{s} ; d t_{1}, \ldots, d t_{s}\right)$.

Théorème 3.1 Soit $\mathcal{O}$ une opérade cofibrante, unitaire, augmentée (l'augmentation est surjective), telle que chaque $R$-module différentiel gradué $\mathcal{O}(l)$ est borné supérieurement.

Alors l'algébre $\Omega^{\mathcal{O}}(X)$ est homotopiquement équivalente à $C^{*} X$ (comme $R$ module différentiel $\mathbb{Z}$-gradué). Cette équivalence d'homotopie est naturelle en $X$.

Algebraic $\mathcal{E} \mathcal{G}$ Geometric Topology, Volume 2 (2002) 
Preuve C'est une conséquence immédiate des lemmes précédents et de la théorie des modèles acycliques.

Remarques On peut en fait montrer que si $\mathcal{O}$ est une opérade unitaire, augmentée (l'augmentation est toujours supposée surjective), telle que chaque $R$ module différentiel gradué $\mathcal{O}(l)$ est borné supérieurement et $R\left[\Sigma_{l}\right]$-projectif, alors le théorème précédent est encore valable. En particulier cette construction s'applique à l'opérade $\mathcal{R}_{B}$. Cette construction s'applique aussi à $\mathcal{A} s$ l'opérade des algèbres associatives. M. Karoubi a aussi défini un foncteur à valeurs dans les algèbres associatives ( $\mathrm{cf}[24],[25])$. Mais ce foncteur diffère de notre foncteur $\Omega^{\mathcal{A} s}$. Enfin si on travaille avec $R$ un corps de caractéristique nulle on peut toujours définir un foncteur de formes différentielles généralisées pour $\mathcal{O}$ une opérade unitaire, augmentée et bornée supérieurement.

\subsection{Théories de cochaînes et structures $\mathcal{E}_{\infty}$}

On fait d'abord quelques rappels sur les notions de théories de cochaînes [43], [27] et de théories cohomologiques [5]. On montre que tout foncteur de formes différentielles généralisées est une théorie de cochaînes. On explore la structure de $\mathcal{E}_{\infty}$-algèbre des théories cohomologiques et des foncteurs de formes différentielles généralisées; on en déduit deux approches pour construire des cup-i produits sur ces objets. Pour finir on montre que le foncteur des formes différentielles généralisées pour les $\mathcal{E}_{\infty}$-algèbres est en un certain sens, universel.

On donne une version $\mathbb{Z}$-graduée des notions de théories de cochaînes et de théories cohomologiques.

Définition 3.3 Soit $F: \mathcal{S}^{o p} \longrightarrow \mathcal{O}-\mathcal{A l} g_{d g}$ un foncteur. On dit que ce foncteur est une théorie de cochaînes s'il satisfait aux propriétés suivantes:

i) Pour tout ensemble simplicial $X$ on a $\pi^{*} F(X) \cong H^{*}(X ; R)$.

ii) Pour toute inclusion simpliciale $i: K \longrightarrow X$, le morphisme induit $F(i): F(X) \longrightarrow F(K)$ est un épimorphisme.

Proposition 3.4 Le foncteur des formes différentielles généralisées $\Omega^{\mathcal{O}}$ définit une théorie de cochaînes.

Preuve La condition i) a été vérifiée dans le paragraphe précédent (théorème 3.1). Vérifions que $\Omega^{\mathcal{O}}$ transforme les inclusions simpliciales en épimorphismes. 
C'est une conséquence immédiate du fait que $\Omega^{\mathcal{O}}$ est un ensemble simplicial contractile. En effet, on considère le diagramme d'ensembles simpliciaux suivants:

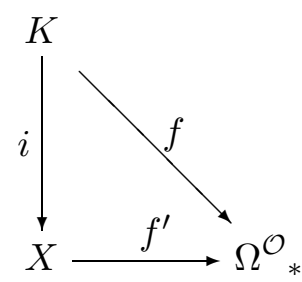

Comme $i$ est une inclusion simpliciale (une cofibration dans la catégorie des ensembles simpliciaux), et que $\Omega^{\mathcal{O}}$ * est contractile et fibrant (comme ensemble simplicial), on peut toujours étendre une application simpliciale $f: K \longrightarrow \Omega^{\mathcal{O}}$ * en un morphisme $f^{\prime}: X \longrightarrow \Omega^{\mathcal{O}}$.

Les axiomes dus à Cartan [5] et à Swan [44] permettent de construire une vaste classe de théories de cochaînes. Nous donnons ici une version $\mathbb{Z}$-graduée de ces axiomes.

Définition 3.4 Soit $M_{*}$ un $R$-module différentiel $\mathbb{Z}$-gradué simplicial et augmenté. On considère le foncteur

$$
\mathcal{M}: \mathcal{S}^{o p} \longrightarrow \mathbf{R} \mathcal{M}_{\mathbf{d g}}
$$

tel que:

$$
\mathcal{M}_{X}=\operatorname{Hom}_{\mathcal{S}}\left(X, M_{*}\right) .
$$

On dit que le foncteur $\mathcal{M}$ vérifie les axiomes de Cartan-Swan s'il satisfait les deux conditions suivantes:

i) Le $R$-module $M_{*}$ est acyclique relativement à l'augmentation. Et le noyau de la différentielle $d: M_{*}^{0} \longrightarrow M_{*}^{1}$ a le type d'homotopie d'un $K(R, 0)$.

ii) Le $R$-module simplicial $M_{*}$ est un ensemble simplicial contractile.

Le théorème suivant est dû à Cartan [5] dans le cas $\mathbb{N}$-gradué; on le généralise au cas $\mathbb{Z}$-gradué:

Théorème 3.2 Si le foncteur $\mathcal{M}$ associé au $R$-module simplicial différentiel $\mathbb{Z}$-gradué $M_{*}$ satisfait les axiomes de Cartan-Swan, alors il définit une théorie de cochaînes. 
Preuve Cette généralisation ne pose pas de difficultés. Rappelons juste les arguments de Cartan.

Soit $Z^{n} M_{*}$ le noyau de la différentielle

$$
d: M_{*}^{n} \longrightarrow M_{*}^{n+1}
$$

Comme le $R$-module $M_{*}$ est acyclique relativement à l'augmentation, on a les suites exactes courtes:

$$
0 \longrightarrow Z^{n} M_{*} \longrightarrow M_{*}^{n} \stackrel{d}{\longrightarrow} Z^{n+1} M_{*} \longrightarrow 0
$$

pour $n \geq 0$.

En tant que suites exactes courtes de groupes abéliens simpliciaux, le morphisme $d$ est une fibration de Kan de fibre $Z^{n} M_{*}$. De plus comme $M_{*}^{n}$ est un ensemble simplicial contractile et que $Z^{0} M_{*}$ a le type d'homotopie d'un $K(R, 0)$, on en déduit que chaque $Z^{n} M_{*}$ a le type d'homotopie d'un $K(R, n)$. Ces suites exactes s'identifient aux fibrations:

$$
K(R, n) \longrightarrow P K(R, n) \longrightarrow K(R, n+1) .
$$

Pour conclure on identifie $\pi^{n} M(X)$ avec $\left[X, Z^{n} M_{*}\right]$.

Les $Z^{n} M_{*}$ forment un spectre dans la catégorie des ensembles simpliciaux (en l'occurence un spectre d'Eilenberg-Mac-Lane $H R$ ).

Proposition 3.5 Le foncteur des formes différentielles généralisées $\Omega^{\mathcal{O}}$ vérifie les axiomes de Cartan-Swan. On a les isomorphismes suivants:

$$
\pi^{n} \Omega^{\mathcal{O}}(X) \cong H^{n}(X, R) \cong\left[X, Z_{n} \Omega^{\mathcal{O}^{*}}\right]
$$

Preuve On vérifie que le noyau $Z_{0} \Omega^{\mathcal{O}}$ * de la différentielle

$$
d: \Omega_{*}^{\mathcal{O}^{0}} \longrightarrow \Omega_{*}^{\mathcal{O}^{1}}
$$

a le type d'homotopie d'un $K(R, 0)$.

Dans la preuve du théorème précédent $\pi_{n} \Omega^{\mathcal{O}}{ }_{X}$ est identifié avec $\left[X, Z_{n} \Omega^{\mathcal{O}}{ }_{*}\right]$, où $Z^{n} \Omega^{\mathcal{O}}$ * est le noyau de

$$
d: \Omega_{*}^{\mathcal{O}^{n}} \longrightarrow \Omega_{*}^{\mathcal{O}^{n+1}}
$$

De plus, on sait que le foncteur des formes différentielles généralisées $\pi^{n} \Omega^{\mathcal{O}}{ }_{X}$ est isomorphe à $H^{n}(X ; R)$.

Ce qui nous permet l'identification de $\pi^{0} \Omega^{\mathcal{O}}(X)$ avec $\left[\mathrm{X}, Z_{0} \Omega^{\mathcal{O}}\right.$ ] . 
On suppose maintenant que $\mathcal{O}=\mathcal{E}_{\infty}$ est un modèle cofibrant de $\mathcal{C}$ om dans la catégorie des opérades unitaires augmentées.

Un des intérêts majeurs des $\mathcal{E}_{\infty}$-algèbres est qu'elles apparaissent de manière naturelle dans l'étude de l'homotopie des espaces topologiques.

En effet, Hinich et Schechtmann [21] ont démontré que pour tout ensemble simplicial $X$, l'algèbre des cochaînes singulières normalisées est munie de manière naturelle d'une structure de $\mathcal{E}_{\infty}$-algèbre.

En coefficients $\mathbb{F}_{p}$, les opérations de Steenrod sur $\pi^{*} C^{*}\left(X ; \mathbb{F}_{p}\right) \cong H^{*}\left(X ; \mathbb{F}_{p}\right)$ déterminées par cette structure $\mathcal{E}_{\infty}$ coincident avec les opérations de Steenrod classiques.

Proposition 3.6 Il existe un morphisme naturel de $\mathcal{E}_{\infty}$-algèbres:

$$
\Omega^{\mathcal{E}_{\infty}}(X) \longrightarrow C^{*}(X ; R)
$$

qui induit une équivalence d'homotopie dans la catégorie $\mathbf{R} \mathcal{M}_{\mathbf{d g}}$.

Preuve On généralise la construction donnée par M. Karoubi dans le cadre des formes différentielles non-commutatives.

Pour ce faire on rappelle qu'une cochaîne singulière $\lambda \in C^{n}(\Delta[s] ; R)$ peut être considérée comme une application qui associe un élément $\lambda\left(i_{0}, \ldots, i_{n}\right)$ de $R$ à toute suite $\left(i_{0}, \ldots, i_{n}\right)$ d'éléments de $\{0, \ldots, s\}$ (ce morphisme doit aussi vérifier des conditions de compatibilité avec les morphismes de faces et de dégénérescences de $\Delta[s]$ ).

Le cup produit

$$
C^{n}(\Delta[s] ; R) \times C^{m}(\Delta[s] ; R) \longrightarrow C^{n+m}(\Delta[s] ; R)
$$

est donné par la formule d'Alexander-Whitney:

$$
(\lambda \cup \mu)\left(i_{0}, \ldots, i_{n+m}\right)=\lambda\left(i_{0}, \ldots, i_{n}\right) \mu\left(i_{n}, \ldots, i_{n+m}\right) .
$$

Si $\lambda \in C^{0}(\Delta[s] ; R)$, son bord $\delta(\lambda)$ est donné par: $\delta(\lambda)(i, j)=\lambda(i)-\lambda(j)$.

Nous allons définir un morphisme de $\mathcal{E}_{\infty}$-algèbres:

$$
\Phi_{s}: \Omega^{\mathcal{E}_{\infty}}(\Delta[s]) \longrightarrow C^{*}(\Delta[s] ; R) .
$$

Comme $\Omega^{\mathcal{E}_{\infty}}(\Delta[s])=\mathcal{E}_{\infty}\left(t_{0}, \ldots, t_{s} ; d t_{0}, \ldots, d t_{s}\right) / I_{s}$, pour définir $\Phi_{s}$ il suffit de donner l'image de $\left\{t_{0}, \ldots, t_{s} d t_{0}, \ldots, d t_{s}\right\}$.

On pose $\Phi_{s}\left(t_{r}\right)=X_{r}$ avec $X_{r} \in R\left[X_{0}, \ldots, X_{s}\right] /\left(\sum_{r=0}^{n} X_{r}=1\right)$ ce polynôme définit un élément de $C^{0}(\Delta[s] ; R)$ : pour $i \in 0, \ldots, s \quad X_{r}(i)$ correspond à l'évaluation de ce polynôme en $(0, \ldots, 0,1,0, \ldots, 0)$ où 1 est en $i^{\text {eme }}$ position. 
On définit $\delta X_{r}$ par $\delta X_{r}(i, j)=X_{r}(i)-X_{r}(j)$, enfin on pose $\Phi_{s}\left(d t_{r}\right)=\delta X_{r}$.

Ces morphismes induisent des quasi-isomorphismes (en effet on remarque que l'image d'un scalaire $r \in R$ par $\Phi_{s}$ est l'application constante de valeur $r$ ).

Tout ce qui précède permet de définir une transformation naturelle:

$$
\Phi: \Omega^{\mathcal{E}_{\infty}} \longrightarrow C^{*}(-; R) .
$$

Et, par un argument de la théorie des modèles acycliques, on en déduit que $\Phi$ induit une équivalence d'homotopie dans la catégorie des $R$-modules différentiels gradués.

On donne maintenant des résultats de comparaison entre les différentes théories de formes différentielles généralisées. Pour ce faire, on remarque que si $\mathcal{O}$ est une opérade cofibrante unitaire augmentée, alors toute $\mathcal{E}_{\infty}$-algèbre est de manière naturelle une $\mathcal{O}$-algèbre; cette structure est induite par le morphisme d'opérades $f$ :

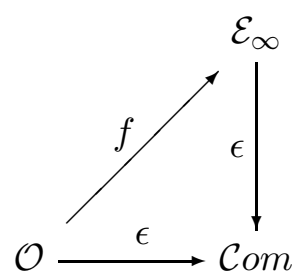

Le morphisme $f$ est un relèvement de l'augmentation $\epsilon$. Celui-ci existe car $\mathcal{O}$ est cofibrante et l'augmentation $\epsilon: \mathcal{E}_{\infty} \longrightarrow \mathcal{C}$ om étant une fibration triviale possède la propriété de relèvement par rapport aux cofibrations.

Proposition 3.7 Soit $\mathcal{O}$ une opérade (cofibrante unitaire augmentée), il existe une structure naturelle de $\mathcal{O}$-algèbre sur $C^{*}(X ; R)$ et sur $\Omega^{\mathcal{E}_{\infty}}(X)$.

De plus, on a des morphismes naturels de $\mathcal{O}$-algèbres:

$$
\begin{gathered}
\Omega^{\mathcal{O}}(X) \longrightarrow \Omega^{\mathcal{E}_{\infty}}(X) \\
\Omega^{\mathcal{O}}(X) \longrightarrow C^{*}(X ; R) .
\end{gathered}
$$

qui induisent des équivalences d'homotopie.

Preuve La structure de $\mathcal{O}$-algèbre sur $C^{*}(X ; R)$ provient du morphisme

$$
f: \mathcal{O} \longrightarrow \mathcal{E}_{\infty}
$$

et du fait que $C^{*}(X ; R)$ est une $\mathcal{E}_{\infty}$-algèbre. 
Le premier morphisme

$$
\Psi_{X}: \Omega^{\mathcal{O}}(X) \longrightarrow \Omega^{\mathcal{E}_{\infty}}(X)
$$

est obtenu via les applications:

$$
\Psi_{s}: \Omega^{\mathcal{O}}(\Delta[s]) \longrightarrow \Omega^{\mathcal{E}_{\infty}}(\Delta[s]) .
$$

On rappelle que:

$$
\Omega^{\mathcal{O}}(\Delta[s])=\mathcal{O}\left(t_{0}, \ldots, t_{s} ; d t_{0}, \ldots, d t_{s}\right) / I_{s}
$$

et que:

$$
\Omega^{\mathcal{E}_{\infty}}(\Delta[s])=\mathcal{E}_{\infty}\left(t_{0}^{\prime}, \ldots, t_{s}^{\prime} ; d t_{0}^{\prime}, \ldots, d t_{s}^{\prime}\right) / I_{s} .
$$

Alors $\Psi_{s}$ est entièrement determinée par les conditions suivantes $\Psi_{s}\left(t_{i}\right)=t_{i}^{\prime}$ et $\Psi_{s}\left(d t_{i}\right)=d t_{i}^{\prime}$.

On donne maintenant deux manières de construire des cup-i produits pour le foncteur $\Omega^{\mathcal{O}}$.

La première méthode applique la théorie des modèles acycliques.

Le groupe symétrique $\Sigma_{p}$ agit sur $\Omega^{\mathcal{O}}(X)^{\otimes p}$ via l'application d'échange $T$.

Soit $\alpha \in \Sigma_{p}$ une permutation cyclique d'ordre $p$. On introduit les opérations suivantes:

$$
\begin{gathered}
\tau=1-\alpha \\
\sigma=1+\alpha+\ldots+\alpha^{p-1} .
\end{gathered}
$$

Elles vérifient $\tau \sigma=\sigma \tau=0$. Considérons le produit $\mu_{0}$ défini par la composée

$$
\mu_{0}: \Omega^{\mathcal{O}}(X)^{\otimes p} \stackrel{s_{p}}{\longrightarrow} \mathcal{O}(p) \otimes \Omega^{\mathcal{O}}(X)^{\otimes p} \stackrel{\theta_{p}}{\longrightarrow} \Omega^{\mathcal{O}}(X)
$$

Avec $s_{p}$ une section de l'augmentation $\epsilon: \mathcal{O}(p) \longrightarrow R$, et $\theta_{p}$ l'action de $\mathcal{O}$ sur $\Omega^{\mathcal{O}}(X)$.

Le morphisme $\mu_{0} \tau$ est nul en homotopie (le produit étant commutatif en homotopie, d'après un argument de la théorie des modèles acycliques). Il existe un homomorphisme $\mu_{1}$ de degré -1 , tel que:

$$
\mu_{0} \tau=\mu_{1} d+d \mu_{1} .
$$

Maintenant on considère le morphisme $\mu_{1} \sigma$ : lui aussi est nul en homotopie. Toujours d'après la théorie des modèles acycliques il existe un homomorphisme $\mu_{2}$, de degré -2 , tel que:

$$
\mu_{1} \sigma=\mu_{2} d-d \mu_{2}
$$


Ainsi par itération on construit une suite d'opérations vérifiant les formules suivantes:

$$
\begin{gathered}
\mu_{2 n} \tau=\mu_{2 n+1} d+d \mu_{2 n+1} \\
\mu_{2 n+1} \sigma=\mu_{2 n} d-d \mu_{2 n} .
\end{gathered}
$$

Si on travaille à coefficients dans $\mathbb{F}_{p}$, les opérations de Steenrod sont obtenues en composant avec la diagonale:

$$
\Omega^{\mathcal{O}}(X) \stackrel{\Delta}{\longrightarrow} \Omega^{\mathcal{O}}(X)^{\otimes p} \stackrel{\mu_{n}}{\longrightarrow} \Omega^{\mathcal{O}}(X) .
$$

Bien sûr toutes ces constructions sont naturelles en $X$.

Décrivons maintenant la seconde approche:

Proposition 3.8 Pour toute opérade cofibrante le foncteur $\Omega^{\mathcal{O}}$ est à valeurs dans la catégorie des $\mathcal{E}_{\infty}$-algèbres.

Preuve On commence par montrer que $\Omega^{\mathcal{O}}(X)$ est une algèbre sur une opérade acyclique que l'on note $\mathcal{E} n d(\mathcal{O})$.

On définit cette opérade par:

$$
\mathcal{E} n d(\mathcal{O})(n)=\operatorname{Hom}_{\mathbf{R} \mathcal{M}_{\mathbf{d g}_{\mathcal{S}}}}\left(\Omega^{\mathcal{O}}{ }^{\otimes n}, \Omega^{\mathcal{O}}{ }_{*}\right)
$$

pour $n \geq 2$, on pose $\mathcal{E} n d(\mathcal{O})(0)=\mathcal{E} n d(\mathcal{O})(1)=R$. Cette opérade est acyclique car on a prouvé que $\Omega^{\mathcal{O}}$ * est contractile comme R-module simplicial différentiel $\mathbb{Z}$-gradué.

Dans la catégorie des opérades il existe un modèle cofibrant noté $\mathcal{E}_{\infty}$ de l'opérade $\mathcal{E} n d(\mathcal{O})$ :

$$
\mathcal{E}_{\infty} \longrightarrow \mathcal{E} n d(\mathcal{O}) \longrightarrow \mathcal{C o m} .
$$

Chaque morphisme est une fibration triviale donc $\mathcal{E}_{\infty}$ est aussi un modèle cofibrant de l'opérade $\mathcal{C}$ om.

Et le morphisme d'opérades $\mathcal{E}_{\infty} \longrightarrow \mathcal{E} n d(\mathcal{O})$ permet de définir une structure de $\mathcal{E}_{\infty}$-algèbre sur $\Omega^{\mathcal{O}}(X)$.

Remarques Le $R$-module $\Omega^{\mathcal{O}}(X)$ est aussi une algèbre sur une seconde opérade notée $T N_{\mathcal{D} \text { old }}(\mathcal{O})$. Cette opérade est telle que:

$$
T N_{\text {Dold }}(\mathcal{O})(n)=\operatorname{Hom}\left(\Omega^{\mathcal{O} \otimes n}, \Omega^{\mathcal{O}}\right)
$$

pour $n \geq 2$, et

$$
T N_{\text {Dold }}(\mathcal{O})(0)=T N_{\text {Dold }}(\mathcal{O})(1)=R .
$$


Ce sont les transformations naturelles entre le foncteur $\Omega^{\mathcal{O}^{\otimes n}}$ et le foncteur $\Omega^{\mathcal{O}}$. L'acyclicité de cette opérade est une conséquence immédiate de la théorie des modèles acycliques. Ceci généralise un résultat de $\mathrm{A}$. Dold [6] obtenu pour le foncteur des chaînes singulières d'un ensemble simplicial. De plus, il existe une paire de morphismes $\phi: T N_{\mathcal{D} \text { old }}(\mathcal{O}) \rightleftharpoons \mathcal{E} n d(\mathcal{O}): \psi$, telle que $\phi$ est une fibration acyclique (dans la catégorie des opérades) et $\phi \psi=I d$.

Soit $T$ un élément de $T N_{\mathcal{D} \text { old }}(\mathcal{O})(n)$, le morphisme $\phi(T) \in \mathcal{E} n d(\mathcal{O})(n)$ est donné par $\phi(T)=T(\Delta)$. On rappelle que $\Delta$ est l'ensemble cosimplicial formé par les $\Delta[p]$.

Considérons $f \in \mathcal{E} n d(\mathcal{O})(n)$, on lui associe la transformation naturelle $T_{f}$ définie comme étant la composée:

$$
T_{f}(X): \operatorname{Hom}_{\mathbf{S}}\left(X, \Omega_{*}^{\mathcal{O}}\right)^{\otimes n} \longrightarrow \operatorname{Hom}_{\mathbf{S}}\left(X, \Omega^{\mathcal{O}}{ }_{*}^{\otimes n}\right) \stackrel{f^{*}}{\longrightarrow} \operatorname{Hom}_{\mathbf{S}}\left(X, \Omega_{*}^{\mathcal{O}}\right) .
$$

Les opérades $T N_{\mathcal{D} \text { old }}(\mathcal{O})$ et $\mathcal{E} n d(\mathcal{O})$ ne sont pas cofibrantes car l'opérade $\mathcal{C}$ om est un rétract de ces deux opérades.

On résume ces résultats par le théorème suivant:

Théorème 3.3 Pour tout ensemble simplicial $X$ l'algèbre $\Omega^{\mathcal{O}}(X)$ est munie de l'action d'opérations. Cette action induit sur $\pi^{*} \Omega^{\mathcal{O}}(X)$ une structure d'algèbre instable sur l'algèbre de Steenrod telle que $\pi^{*} \Omega^{\mathcal{O}}(X) \cong H^{*}\left(X, \mathbb{F}_{p}\right)$ soit un isomorphisme d'algèbres instables.

La proposition suivante relie les différents foncteurs de formes différentielles généralisées.

Proposition 3.9 i) Il existe un morphisme naturel de $\mathcal{E}_{\infty}$-algèbres:

$$
\Omega^{\mathcal{E}_{\infty}}(X) \longrightarrow \Omega^{\mathcal{O}}(X)
$$

qui induit une équivalence d'homotopie dans la catégorie $\mathbf{R} \mathcal{M}_{\mathbf{d g}}$.

ii) Si $R$ est un corps de caractéristique nulle, on note $\mathcal{A}_{P L}$ le foncteur de Sullivan ([43], [4], [13]). Alors on a un morphisme naturel de $\mathcal{E}_{\infty}$-algèbres:

$$
\Omega^{\mathcal{E}_{\infty}}(X) \longrightarrow \mathcal{A}_{P L}(X)
$$

qui induit une équivalence d'homotopie dans la catégorie $\mathbf{R} \mathcal{M}_{\mathbf{d g}}$.

iii) Si on note $\Omega_{\mathcal{K}}$ le foncteur des formes différentielles non-commutatives introduit par M. Karoubi [24], [25], on a un morphisme naturel de $\mathcal{E}_{\infty}$-algèbres:

$$
\Omega^{\mathcal{E}_{\infty}}(X) \longrightarrow \Omega_{\mathcal{K}}(X)
$$

qui induit une équivalence d'homotopie dans la catégorie $\mathbf{R} \mathcal{M}_{\mathbf{d g}}$.

Preuve Comme pour les propositions précédentes, on peut donner des formules explicites pour chacun de ces morphismes. 


\subsection{Applications à l'homotopie des algèbres sur une opérade}

On suppose que l'opérade $\mathcal{O}$ permet de définir un foncteur de formes différentielles généralisées. Dans ce cadre on va généraliser quelques constructions bien connues en homotopie rationnelle. En fait on retrouve les constructions de l'homotopie rationnelle, quand on prend $R=\mathbb{Q}$ et $\mathcal{O}=\mathcal{C}$ om , le foncteur $\Omega^{\mathcal{C o m}}$ est le foncteur $A_{P L}$ de Sullivan ([43],[4]).

Définition 3.5 Pour deux $\mathcal{O}$-algèbres $\mathrm{A}$ et $\mathrm{B}$ on définit un espace fonctionnel simplicial $\operatorname{Hom}^{\Delta}(A, B)$ dont les n-simplexes sont donnés par:

$H_{o m}{ }^{\Delta n]}(A, B)=H_{o m} m_{\mathcal{A} \lg _{\mathrm{dg}}}\left(A, \mathcal{O}^{n} \amalg B\right)$.

Définition 3.6 Le foncteur

$$
\mathbb{M}: \mathcal{O}-\mathcal{A l} g_{d g}^{o p} \longrightarrow \mathcal{S}
$$

donné par:

$$
\mathbb{M}(A)=\operatorname{Hom}_{\mathcal{O}-\mathcal{A} \lg _{\mathrm{dg}}}\left(A, \Omega_{*}^{\mathcal{O}}\right)=\operatorname{Hom}^{\Delta}(A, R) .
$$

est appelé foncteur de réalisation simpliciale.

Proposition 3.10 Les foncteurs contravariants $\Omega^{\mathcal{O}}$ et $\mathbb{M}$ sont des foncteurs adjoints au sens de Quillen entre les catégories $\mathcal{O}-\mathcal{A} \lg _{\mathrm{dg}}$ et $\mathcal{S}$.

Preuve On commence par montrer que $\Omega^{\mathcal{O}}$ et $\mathbb{M}$ sont adjoints, c'est-à dire que l'on a une bijection naturelle

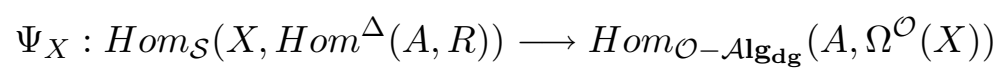

Si $X=\Delta[n]$ c'est évident (d'après la définition du foncteur $\mathbb{M}$ ).

Tout ensemble simplicial peut s'écrire sous la forme d'un coégalisateur:

$$
\amalg_{s \in S} \Delta[s] \underset{g}{\stackrel{f}{\longrightarrow}} \amalg_{t \in T} \Delta[t] \longrightarrow X
$$

On utilise la propriété suivante du foncteur $\Omega^{\mathcal{O}}$ :

Le foncteur $\Omega^{\mathcal{O}}: \mathcal{S} \longrightarrow \mathcal{O}-\mathcal{A} \mathbf{l g}_{\text {dg }}$ transforme les colimites en limites. D'où en appliquant $\Omega^{\mathcal{O}}$ au coégalisateur précédent on obtient l'égalisateur:

$$
\Omega^{\mathcal{O}}(X) \longrightarrow \Omega^{\mathcal{O}}\left(\amalg_{t \in T} \Delta[t]\right) \underset{\overrightarrow{\Omega^{\mathcal{O} g}}}{\stackrel{\Omega^{\mathcal{O} f}}{\longrightarrow}} \Omega^{\mathcal{O}}\left(\amalg_{s \in S} \Delta[s]\right)
$$


Puis

$$
\Omega^{\mathcal{O}}(X) \longrightarrow \prod_{t \in T} \Omega^{\mathcal{O}}(\Delta[t]) \rightrightarrows \prod_{s \in S} \Omega^{\mathcal{O}}(\Delta[s])
$$

et

$$
\Omega^{\mathcal{O}}(X) \amalg R \rightarrow \prod_{t \in T}\left(\Omega^{\mathcal{O}}(\Delta[t]) \amalg R\right) \rightrightarrows \prod_{s \in S}\left(\Omega^{\mathcal{O}}(\Delta[s]) \amalg R\right)
$$

Le foncteur $\operatorname{Hom}_{\mathcal{O}-\mathcal{A l g}} \lg _{\mathrm{dg}}(A,-)$ transforme les produits en produits et les égalisateurs en égalisateurs. On a alors:

$$
\begin{array}{r}
H \operatorname{Hom}_{\mathcal{O}-\mathcal{A} \lg _{\mathrm{dg}}}\left(A, \Omega^{\mathcal{O}}(X) \amalg R\right) \longrightarrow \prod_{t \in T} \operatorname{Hom}_{\mathcal{O}-\mathcal{A l g} \mathrm{dg}}\left(A, \Omega^{\mathcal{O}}(\Delta[t]) \amalg R\right) \\
\ldots \\
\ldots \prod_{s \in S} \operatorname{Hom}_{\mathcal{O}_{-} \mathcal{A l g d g}}\left(A, \Omega^{\mathcal{O}}(\Delta[s]) \amalg R\right)
\end{array}
$$

Si on applique le foncteur $\operatorname{Hom}_{\mathcal{S}}\left(-, \operatorname{Hom}^{\Delta}(A, B)\right)$ au coégalisateur initial, on obtient l'égalisateur:

$$
\begin{array}{r}
\operatorname{Hom}_{\mathcal{S}}\left(X, \operatorname{Hom}^{\Delta}(A, R)\right) \longrightarrow \prod_{t \in T} \operatorname{Hom}_{\mathcal{S}}\left(\Delta[t], \operatorname{Hom}^{\Delta}(A, R)\right) \\
\ldots \rightleftharpoons \prod_{s \in S} \operatorname{Hom}_{\mathcal{S}}\left(\Delta[s], \operatorname{Hom}^{\Delta}(A, R)\right)
\end{array}
$$

Comme $\Psi$ est une bijection sur les seconds et troisièmes termes, c'est aussi une bijection sur les premiers.

Pour finir, on remarque que le foncteur $\Omega^{\mathcal{O}}$ transforme les cofibrations entre ensembles simpliciaux en fibrations et qu'il préserve les équivalences faibles.

Grâce à la notion de formes différentielles généralisées, on peut définir un objet chemin pour la catégorie des $\mathcal{O}$-algèbres: en effet considérons la $\mathcal{O}$-algèbre $\Omega_{1}^{\mathcal{O}}=\Omega^{\mathcal{O}}(\Delta[1])$ (avec $\Delta[1]$ le 1-simplexe standard). Puis on définit le foncteur:

$$
-\amalg \Omega_{1}^{\mathcal{O}}: \mathcal{O}-\mathcal{A} \lg _{\mathrm{dg}} \longrightarrow \mathcal{O}-\mathcal{A} \lg _{\mathrm{dg}}
$$

Pour toute $\mathcal{O}$-algèbre $A$ on a les applications suivantes:

$$
A \stackrel{p}{\longrightarrow} A \amalg \Omega_{1}^{\mathcal{O}} \stackrel{\left(d_{0}, d_{1}\right)}{\longrightarrow} A \times A .
$$

On rappelle que l'on peut identifier $\Omega_{1}^{\mathcal{O}}$ avec $\mathcal{O}(t, d t)$, on a des morphismes

$$
\delta_{0}, \delta_{1}: \Omega_{1}^{\mathcal{O}} \longrightarrow \Omega_{0}^{\mathcal{O}} \cong R
$$

tels que $\delta_{i}(d t)=0$ et $\delta_{i}(t)=i$. Le morphisme $\mathrm{p}$ est l'application canonique. Et les applications $d_{0}, d_{1}$ sont définies comme étant l'identité sur $A$ et $\delta_{i}$ sur $\Omega_{1}^{\mathcal{O}}$. 
Ces morphismes satisfont les propriétés suivantes:

i) $d_{i} p$ est l'identité, $i=0,1$.

ii) $d_{i}$ est une fibration triviale, $i=0,1$. Le morphsime $\left(d_{0}, d_{1}\right)$ est une fibration.

iii) $\quad p$ est une équivalence faible.

Grâce à ces observations on définit maintenant une notion d'homotopie simpliciale.

Définition 3.7 Deux morphismes $f, g: A \longrightarrow B$ sont simplicialement homotopes s'il existe une application $\mathrm{H}$ de $\operatorname{Hom}^{\Delta[1]}(A, B), H: A \longrightarrow B \amalg \Omega_{1}^{\mathcal{O}}$ telle que $d_{0} H=f$ et $d_{1} H=g$.

Proposition 3.11 L'homotopie simpliciale satisfait les propriétés suivantes:

i) Soient deux applications $f, g: A \longrightarrow B$ qui sont simplicialement homotopes, alors:

$$
\pi^{*} f=\pi^{*} g: \pi^{*} A \longrightarrow \pi^{*} B .
$$

ii) Si $A$ est une $\mathcal{O}$-algèbre cofibrante l'homotopie simpliciale est une relation d'équivalence.

iii) Si $A$ est cofibrante on a l'isomorphisme:

$$
[A, B]_{H o O}-\mathcal{A l g} \mathbf{g}_{\mathrm{dg}} \cong \pi^{0} \operatorname{Hom}^{\Delta}(A, B) .
$$

\section{Un modèle de la suite spectrale de Leray-Serre}

Un des outils fondamentaux en homotopie rationnelle est la construction du modèle algébrique de la fibre à partir de celui de la fibration. Construction que l'on doit à P.P. Grivel [17] (pour les espaces 1-connexes) et à S. Halperin [18] (pour les espaces nilpotents).

Nous généralisons l'approche de P.P. Grivel pour le foncteur $\mathcal{A}_{P L}$ de Sullivan aux formes différentielles généralisées pour les $\mathcal{O}$-algèbres.

Les quatre premiers paragraphes sont consacrés à la construction d'une suite spectrale de Leray-Serre au moyen des formes différentielles généralisées. A cet effet, on définit des formes différentielles généralisées avec coefficients locaux (paragraphe 1), puis des formes différentielles généralisées pour les bisimplexes (paragraphe 2). 
Les techniques bisimpliciales (Dress [8],[29]) sont utilisées pour associer un bicomplexe à une fibration (paragraphe 3 ). En filtrant ce bicomplexe, on retrouve la suite spectrale de Leray-Serre (paragraphe 4.3, théorème 4.1).

Dans le paragraphe 4.4, on construit une suite spectrale à partir du modèle cofibrant en $\mathcal{E}_{\infty}$-algèbres d'une fibration. Par un théorème de comparaison entre cette nouvelle suite spectrale et la suite spectrale de Leray-Serre construite précédemment, on en déduit un modèle de la fibre (proposition 4.9).

Le paragraphe 4.5 donne une interprétation algébrique (sur le modèle) de la transgression pour la suite spectrale de Leray-Serre.

\subsection{Préfaisceaux sur les ensembles simpliciaux}

Soit $X$ un ensemble simplicial; on lui associe une catégorie que l'on note $\mathbf{X}$. Les objets de $\mathbf{X}$ sont les $p$-simplexes de $X$. Les morphismes de $\mathbf{X}$ correspondent aux applications $\alpha: \Delta[p] \longrightarrow \Delta[q]$ telles que le diagramme ci-dessous commute:

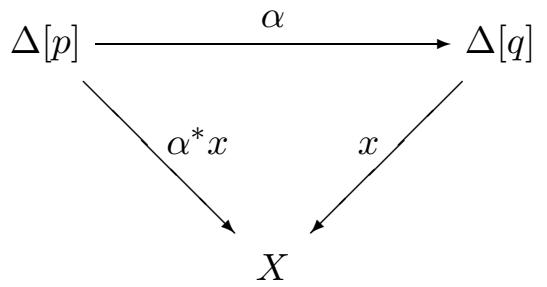

A toute application simpliciale $f: X \longrightarrow Y$ est associé un foncteur $\mathbf{f}: \mathbf{X} \longrightarrow$ Y.

Un préfaisceau sur $X$ à valeurs dans une catégorie $\mathcal{C}$ est un foncteur contravariant de $\mathbf{X}$ dans $\mathcal{C}$. On note $\mathcal{F}(\alpha, x): \mathcal{F}\left(\alpha^{*} x\right) \longrightarrow \mathcal{F}(x)$ l'application induite par $\alpha: \Delta[p] \longrightarrow \Delta[q]$.

Les préfaisceaux que l'on considère sont à valeurs dans $\mathbf{A b}$ (la catégorie des groupes abéliens), dans $\mathbf{R} \mathcal{M}_{\mathrm{dg}}$ ou dans $\mathcal{O}-\mathcal{A} l g_{d g}$.

Si $f: X \longrightarrow Y$ est une application simpliciale et si $\mathcal{F}$ est un préfaisceau sur $X$ alors on a un préfaisceau image réciproque $f^{*} \mathcal{F}=\mathcal{F} \mathbf{f}$.

Les préfaisceaux sur $X$ à valeurs dans une catégorie abélienne forment encore une catégorie abélienne. Une suite de préfaisceaux

$$
0 \longrightarrow \mathcal{F} \longrightarrow \mathcal{G} \longrightarrow \mathcal{H} \longrightarrow 0
$$

est exacte si pour tout $p$-simplexe $x_{p}$ de $X$ la suite

$$
0 \longrightarrow \mathcal{F}\left(x_{p}\right) \longrightarrow \mathcal{G}\left(x_{p}\right) \longrightarrow \mathcal{H}\left(x_{p}\right) \longrightarrow 0
$$


est exacte.

Les foncteurs formes différentielles généralisées sont des préfaisceaux. Les cochaînes singulières sont aussi un exemple de préfaisceau.

Préfaisceaux simpliciaux Un exemple important est le cas des préfaisceaux à valeurs dans $\mathbf{S C}$ la catégorie des objets simpliciaux de $\mathbf{C}$. Soit $\mathcal{F}$ un préfaisceau à valeurs dans $\mathbf{S C}$.

Une section $s$ du préfaisceau $\mathcal{F}$ est la donnée pour tout $q$-simplexe $x \in X_{q}$ d'un ensemble simplicial $s(x)$ tel que pour toute application $\alpha:[p] \longrightarrow[q]$ on a:

$$
\mathcal{F}(\alpha, x)\left(s\left(\alpha^{*}(x)\right)\right)=\alpha^{*}(s(x)) .
$$

Un préfaisceau constant $\mathcal{F}$ sur $X$ est tel que pour tout $q$-simplexe $x \in X_{q}$, $\mathcal{F}(x)=M$ avec $M$ un objet de SC; une section de $\mathcal{F}$ est une application simpliciale de $X$ dans $M$.

Systèmes de coefficents locaux Un système de coefficients locaux $\mathcal{L}$ sur un ensemble simplicial $X$ est un préfaisceau sur $X$ à valeurs dans $\mathbf{A b}$ tel que pour tout opérateur de face $\delta_{i}: \Delta[p+1] \longrightarrow \Delta[p]$ et pour tout $p$-simplexe $x \in X_{p}$, les morphismes $\mathcal{L}\left(\delta_{i} ; x\right): \mathcal{L}\left(\delta_{i} x\right) \longrightarrow \mathcal{L}(x)$ soient des isomorphismes.

Pour tout 0 -simplexe $x \in X$, on a une action de $\pi_{1}(X, x)$ sur le groupe abélien $\mathcal{L}(x)$. Le système de coefficients est dit simple si l'action des groupes fondamentaux est triviale. De plus si $X$ est connexe et si le système de coefficients est simple alors les groupes $\mathcal{L}(x)$ sont isomorphes.

On construit un foncteur $\Omega^{\mathcal{O}}(X ;-): \mathbf{F R} \mathcal{M}_{\mathbf{d g}_{\mathbf{X}}} \longrightarrow \mathbf{S F R} \mathcal{M}_{\mathbf{d g}_{\mathbf{X}}}$ de la catégorie des préfaisceaux sur $X$ à valeurs dans $\mathbf{R} \mathcal{M}_{\mathbf{d g}}$ vers la catégorie des préfaisceaux sur $X$ à valeurs dans $\mathbf{S R} \mathcal{M}_{\mathbf{d g}}$.

On fixe $\mathcal{F}$ un préfaisceau sur $X$ à valeurs dans $\mathbf{R} \mathcal{M}_{\mathbf{d g}}$. On lui associe un préfaisceau noté $\Omega^{\mathcal{O}}(X ; \mathcal{F})$ sur $X$ à valeurs dans $\mathbf{S R} \mathcal{M}_{\mathbf{d g}}$. Ce préfaisceau associe à $x \in X$ le module différentiel gradué simplicial $\Omega_{*}^{\mathcal{O}} \otimes \mathcal{F}(x)$.

Définition 4.1 Une section du préfaisceau sur $\Omega^{\mathcal{O}}(X ; \mathcal{F})$ s'appelle une forme différentielle généralisée sur $X$ à valeurs dans le préfaisceau $\mathcal{F}$. On note $\underline{\Omega}^{\mathcal{O}}(X ; \mathcal{F})$ l'ensemble de ses sections.

L'objet $\underline{\Omega}^{\mathcal{O}}(X ; \mathcal{F})$ est naturellement bigradué. L'ensemble des éléments de bidegré $(r, s)$ est noté $\underline{\Omega}^{\mathcal{O}, r}\left(X ; \mathcal{F}^{s}\right)$. Le degré $s$ est donné par le degré différentiel de $\mathcal{F}$, et le degré $r$ par celui de $\Omega_{*}^{\mathcal{O}}$. On a donc bien un foncteur $\underline{\Omega}^{\mathcal{O}, *}\left(X ; \mathcal{F}^{*}\right)$ de la catégorie des ensembles simpliciaux vers la catégorie des $R$-modules différentiels bigradués. 
Intéressons-nous au cas où $\mathcal{F}$ est un préfaisceau constant. Alors $\mathcal{F}$ s'identifie à un R-module différentiel gradué $M$ concentré en degré 0 . Une forme différentielle généralisée à valeurs dans $\mathcal{F}$ est une application simpliciale de $X$ dans $\Omega_{*}^{\mathcal{O}} \otimes M$. En particulier si $M=R$ alors on a $\underline{\Omega}^{\mathcal{O}}(X ; R)=\Omega^{\mathcal{O}}(X)$.

Proposition 4.1 Soit $X$ un ensemble simplicial et $\mathcal{F}=M$ un préfaisceau constant, avec $M$ un $R$-module plat; alors on a l'isomorphisme:

$$
\pi^{*} \underline{\Omega}^{\mathcal{O}}(X ; M) \cong H^{*}(X ; M) .
$$

Preuve On utilise la théorie des modèles acycliques. Le foncteur $\underline{\Omega}^{\mathcal{O}}(-; M)$ est coreprésentable car l'ensemble simplicial $\Omega_{*}^{\mathcal{O}} \otimes M$ est contractile.

Le foncteur est acyclique car pour tout $p$ le $R$-module différentiel gradué $\Omega^{\mathcal{O}}(\Delta[p]) \otimes M$ est acyclique.

De plus le foncteur $\underline{\Omega}^{\mathcal{O}}(X ;-)$ est exact:

Proposition 4.2 Si $0 \longrightarrow \mathcal{F} \longrightarrow \mathcal{G} \longrightarrow \mathcal{H} \longrightarrow 0$ est une suite exacte de préfaisceaux, alors la suite

$$
0 \longrightarrow \Omega^{\mathcal{O}}(X ; \mathcal{F}) \longrightarrow \Omega^{\mathcal{O}}(X ; \mathcal{G}) \longrightarrow \Omega^{\mathcal{O}}(X ; \mathcal{H}) \longrightarrow 0
$$

est exacte, tout comme la suite

$$
0 \longrightarrow \underline{\Omega}^{\mathcal{O}}(X ; \mathcal{F}) \longrightarrow \underline{\Omega}^{\mathcal{O}}(X ; \mathcal{G}) \longrightarrow \underline{\Omega}^{\mathcal{O}}(X ; \mathcal{H}) \longrightarrow 0 .
$$

Preuve On suppose que $0 \longrightarrow \mathcal{F} \stackrel{u}{\longrightarrow} \mathcal{G} \stackrel{v}{\longrightarrow} \mathcal{H} \longrightarrow 0$ est une suite exacte de préfaisceaux sur un ensemble simplicial $X$ ce qui signifie que pour tout $x \in X$ la suite

$$
0 \longrightarrow \mathcal{F}(x) \stackrel{u_{x}}{\longrightarrow} \mathcal{G}(x) \stackrel{v_{x}}{\longrightarrow} \mathcal{H}(x) \longrightarrow 0
$$

est exacte. La platitude de $\Omega(\Delta)$ entraîne que

$$
0 \longrightarrow \Omega_{*}^{\mathcal{O}} \otimes \mathcal{F}(x) \stackrel{I d \otimes u_{x}}{\longrightarrow} \Omega_{*}^{\mathcal{O}} \otimes \mathcal{G}(x) \stackrel{I d \otimes v_{x}}{\longrightarrow} \Omega_{*}^{\mathcal{O}} \otimes \mathcal{H}(x) \longrightarrow 0
$$

est une suite exacte courte. Donc

$$
0 \longrightarrow \Omega_{*}^{\mathcal{O}} \otimes \mathcal{F} \stackrel{I d \otimes u}{\longrightarrow} \Omega_{*}^{\mathcal{O}} \otimes \mathcal{G} \stackrel{I d \otimes v}{\longrightarrow} \Omega_{*}^{\mathcal{O}} \otimes \mathcal{H} \longrightarrow 0
$$

est aussi une suite exacte courte.

L'exactitude de la suite

$$
0 \longrightarrow \underline{\Omega}^{\mathcal{O}}(X ; \mathcal{F}) \longrightarrow \underline{\Omega}^{\mathcal{O}}(X ; \mathcal{G}) \longrightarrow \underline{\Omega}^{\mathcal{O}}(X ; \mathcal{H}) \longrightarrow 0 .
$$

se déduit de la propriété d'extension des formes différentielles. 
Soit $\mathcal{F}$ est un préfaisceau sur $X$ à valeurs dans $\mathbf{R} \mathcal{M}_{\mathbf{d g}}$. On note $d$ sa différentielle, $\mathcal{Z}^{s}$ le préfaisceau des cobords de degré $s$ de $\mathcal{F}$ et $\mathcal{H}^{s}(\mathcal{F})$ le préfaisceau des classes de cohomologie de degré $s$ de $\mathcal{F}$. De la suite exacte:

$$
0 \longrightarrow \mathcal{Z}^{s} \longrightarrow \mathcal{F}^{s} \longrightarrow \mathcal{H}^{s}(\mathcal{F}) \longrightarrow 0
$$

et de la proposition précédente on déduit le résultat suivant:

Corollaire 4.1 On a $\pi^{s}\left(\underline{\Omega}^{\mathcal{O}}(X ; \mathcal{F})\right) \cong \underline{\Omega}^{\mathcal{O}}\left(X ; \mathcal{H}^{s}(\mathcal{F})\right)$ où $\pi^{s}\left(\underline{\Omega}^{\mathcal{O}}(X ; \mathcal{F})\right)$ est la cohomologie de $\underline{\Omega}^{\mathcal{O}}(X ; \mathcal{F})$ calculée avec la différentielle de $\mathcal{F}$.

\subsection{Les formes différentielles généralisées pour les bisimplexes}

Le $R$-module différentiel $\Omega_{*}^{\mathcal{O}} \otimes \Omega_{*}^{\mathcal{O}}$ est un $R$-module différentiel bisimplicial et bigradué, un élément de bidimension $(p, q)$ est un élément de $\Omega^{\mathcal{O}}(\Delta[p]) \otimes$ $\Omega^{\mathcal{O}}(\Delta[q])$. On note $\Omega_{b i}^{\mathcal{O}}(X)$ l'ensemble des formes différentielles généralisées pour le bisimplexe $X, \Omega_{b i}^{\mathcal{O}}(X)=\operatorname{Hom}_{B i \mathcal{S}}\left(X, \Omega_{*}^{\mathcal{O}} \otimes \Omega_{*}^{\mathcal{O}}\right)$.

Une forme différentielle généralisée de bidimension $(p, q)$ sur un ensemble bisimplicial $X$ est une application bisimpliciale de $X$ dans $\Omega_{p}^{\mathcal{O}} \otimes \Omega_{q}^{\mathcal{O}}$.

L'objet $\Omega_{*}^{\mathcal{O}} \otimes \Omega_{*}^{\mathcal{O}}$ possède une autre bigraduation donnée par le degré différentiel. Une forme différentielle généralisée de bidegré $(l, m)$ sur $X$ un ensemble bisimplicial est une application bisimpliciale de $X$ dans $\Omega_{*}^{\mathcal{O}, l} \otimes \Omega_{*}^{\mathcal{O}, m}$.

On a un foncteur $\beta: \mathcal{S} \longrightarrow B i \mathcal{S}$ de la catégorie des ensembles simpliciaux dans la catégorie des ensembles bisimpliciaux tel que:

$$
(\beta X)_{p, q}=X_{p}
$$

pour tout $q$. On vérifie aisément que pour tout ensemble simplicial $X$ on a:

$$
\Omega_{b i}^{\mathcal{O}}(\beta X)=\Omega^{\mathcal{O}}(X) .
$$

Soit $f: E \longrightarrow B$ une surjection simpliciale. A ce morphisme on associe un ensemble bisimplicial $S_{., .} f$. Un élément de $S_{p, q} f$ est un couple d'applications $w: \Delta[p] \times \Delta[q] \longrightarrow E$ et $u: \Delta[p] \longrightarrow B$ telles que:

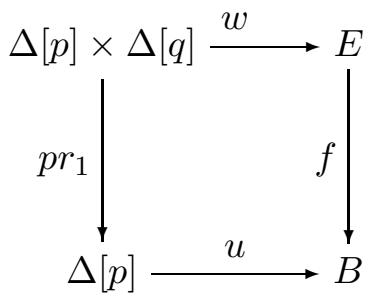

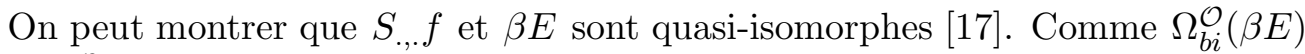
$=\Omega^{\mathcal{O}}(E)$ on en déduit la proposition: 
Proposition 4.3 Soit $f: E \longrightarrow B$ une surjection simpliciale. On a l'isomorphisme:

$$
\pi^{*}\left(\Omega_{b i}^{\mathcal{O}}\left(S_{., .} f\right)\right) \cong H^{*}(E ; R)
$$

Le bisimplexe $S_{\ldots} f$ permet de définir un système de coefficients locaux sur $B$. On considère $B$ comme une catégorie (les objets de $B$ sont les p-simplexes) et on définit un foncteur $\mathbb{F}: B \longrightarrow \mathcal{S}$ en associant à tout simplexe $u_{p}: \Delta[p] \longrightarrow B$ l'ensemble simplicial:

$$
\left(\mathbb{F}\left(u_{p}\right)\right)_{q}=\left\{w_{p, q} /\left(w_{p, q}, u_{p}\right) \in S_{p, q} f\right\}
$$

On a alors le système de coefficients locaux $\mathcal{F}: B \longrightarrow \mathbf{R} \mathcal{M}_{\mathbf{d g}}$ en posant $\mathcal{F}\left(u_{p}\right)=\Omega^{\mathcal{O}}\left(\mathbb{F}\left(u_{p}\right)\right)$. On a un préfaisceau $\Omega^{\mathcal{O}}(B, \mathcal{F})$ sur $B$ à valeurs dans $\mathcal{F}$.

Proposition 4.4 On a $H^{*}\left(\underline{\Omega}^{\mathcal{O}, r}\left(B ; \mathcal{F}^{s}\right) ; d_{\mathcal{F}}\right) \cong \underline{\Omega}^{\mathcal{O}, r}\left(B ; \mathcal{H}^{s}(\mathcal{F})\right)$. De plus si $f: E \longrightarrow B$ est une fibration de Kan et si le système de coefficients locaux $\mathcal{F}$ est simple, alors on a aussi:

$$
H^{*}\left(\underline{\Omega}^{\mathcal{O}, r}\left(B ; \mathcal{F}^{s}\right) ; d_{\mathcal{F}}\right) \cong \underline{\Omega}^{\mathcal{O}, r}\left(B ; H^{s}(\mathcal{F})\right) .
$$

\subsection{Formes différentielles et suites spectrales de Leray-Serre}

Dans ce paragraphe on donne une construction de la suite spectrale de LeraySerre à partir des formes différentielles généralisées.

On utilise cette suite spectrale dans les paragraphes suivants dans le cadre des algèbres sur une $E_{\infty}$-opérade cofibrante. Ceci permet de donner un modèle algébrique des fibrations.

Théorème 4.1 Soit $f: E \longrightarrow B$ une application surjective entre ensembles simpliciaux (on suppose que la cohomologie de $B$ ou que $\mathcal{H}^{s}(\mathcal{F})$ sont finis).

i) On a une suite spectrale qui converge vers $H^{*}(E ; R)$, telle que:

$E_{0}^{r, s}=\underline{\Omega}^{\mathcal{O}, r}\left(B ; \mathcal{F}^{s}\right), E_{1}^{r, s}=\underline{\Omega}^{\mathcal{O}, r}\left(B ; \mathcal{H}^{s}(\mathcal{F})\right)$ et $E_{2}^{r, s}=H^{r}\left(B ; \mathcal{H}^{s}(\mathcal{F})\right)$

où $\mathcal{F}$ est le système de coefficients locaux sur $B$ défini précédemment.

ii) On suppose que $f$ est une fibration de Kan, que $B$ est connexe et que le système de coefficients locaux $\mathcal{H}^{s}(\mathcal{F}$ ) est simple (ce qui est le cas si $B$ est 1-connexe). Alors

$$
E_{2}^{r, s}=H^{r}\left(B ; H^{s}(F ; R)\right)
$$

$F$ désignant la fibre de $f$. 
Preuve On considère la première filtration du bicomplexe $\Omega_{b i}^{\mathcal{O}}\left(S_{r, s} f\right)$. Une forme $\omega \in \Omega_{b i}^{\mathcal{O}}\left(S_{r, s} f\right)$ est de filtration $n$, si pour tout bisimplexe $(w, u) \in S_{p, q} f$ on a $\omega(w, u) \in \bigoplus_{r \geq n} \Omega_{p}^{\mathcal{O}, r} \otimes \Omega_{q}^{\mathcal{O}}$.

De cette filtration on déduit une suite spectrale telle que: $E_{0}^{r, s}=\underline{\Omega}^{\mathcal{O}, r}\left(B ; \mathcal{F}^{s}\right)$. En fait on explicite deux morphismes de modules différentiels gradués:

$$
\phi: \underline{\Omega}^{\mathcal{O}, r}\left(B ; \mathcal{F}^{s}\right) \rightleftharpoons \Omega_{b i}^{\mathcal{O}}\left(S_{r, s} f\right): \psi
$$

inverses l'un de l'autre.

L'application $\phi$ est définie de la manière suivante.

Soit $\omega \in \underline{\Omega}^{\mathcal{O}, r}\left(B ; \mathcal{F}^{s}\right)$ et $u_{p}$ un $p$-simplexe de $B$ on a

$$
\omega\left(u_{p}\right) \in \Omega_{p}^{\mathcal{O}, r} \otimes \mathcal{F}^{s}\left(u_{p}\right)
$$

et par définition

$$
\Omega_{p}^{\mathcal{O}, r} \otimes \mathcal{F}^{s}\left(u_{p}\right)=\Omega_{p}^{\mathcal{O}, r} \otimes \Omega^{\mathcal{O}, s}\left(\mathbb{F}\left(u_{p}\right)\right)=\underline{\Omega}^{\mathcal{O}, s}\left(\mathbb{F}\left(u_{p}\right) ; \Omega_{p}^{\mathcal{O}, r}\right) .
$$

L'application bisimpliciale $\phi(\omega)$ envoie $\left(w_{p, q}, u_{p}\right) \in S_{p, q} f$ sur $\omega\left(u_{p}\right)\left(w_{p, q}\right)$.

A l'inverse, soit $\omega \in \Omega_{b i}^{\mathcal{O}}\left(S_{r, s} f\right)$ et $u_{p}$ un $p$-simplexe de $B$, alors pour $w_{p, q} \in$ $\left(\mathbb{F}\left(u_{p}\right)\right)_{q}$ on pose

$$
\psi(\omega)\left(u_{p}\right)\left(w_{p, q}\right)=\omega\left(w_{p, q}, u_{p}\right)
$$

Comme la différentielle de $E_{0}^{r, s}$ est induite par la deuxième différentielle de $\Omega_{b i}^{\mathcal{O}}\left(S_{r, s} f\right)$, elle s'identifie à la différentielle $d_{\mathcal{F}}$ sur $\underline{\Omega}^{\mathcal{O}, r}\left(B ; \mathcal{F}^{s}\right)$. On a donc l'isomorphisme:

$$
H^{*}\left(\underline{\Omega}^{\mathcal{O}, r}\left(B ; \mathcal{F}^{s}\right) ; I d \otimes d\right) \cong \underline{\Omega}^{\mathcal{O}, r}\left(B ; \mathcal{H}^{s}(\mathcal{F})\right)
$$

d'où:

$$
E_{1}^{r, s}=\underline{\Omega}^{\mathcal{O}, r}\left(B ; \mathcal{H}^{s}(\mathcal{F})\right)
$$

et par suite:

$$
E_{2}^{r, s}=H^{r}\left(B ; \mathcal{H}^{s}(\mathcal{F})\right)
$$

\subsection{Modèle d'une fibration}

On suppose maintenant que l'opérade $\mathcal{O}=\mathcal{E}_{\infty}$ est un modèle cofibrant de l'opérade $\mathcal{C}$ om. L'existence de la suite spectrale de Leray-Serre associée aux formes différentielles généralisées pour les $\mathcal{E}_{\infty}$-algèbres nous permet de décrire un modèle algébrique des fibrations. Au préalable nous avons besoin de résultats concernant le coproduit des $\mathcal{E}_{\infty}$-algèbres. 
Le produit tensoriel des opérades Soient $\mathcal{P}$ et $\mathcal{Q}$ deux opérades. On définit l'opérade $\mathcal{P} \otimes \mathcal{Q}$ en posant $(\mathcal{P} \otimes Q)(n)=\mathcal{P}(n) \otimes Q(n)$. On remarque que si $A$ est une $P$-algèbre et si $B$ est une $Q$-algèbre alors $A \otimes B$ est une $P \otimes Q$-algèbre.

\section{Le coproduit des $\mathcal{E}_{\infty}$-algèbres}

Proposition 4.5 Il existe un morphisme d'opérades $\Delta: \mathcal{E}_{\infty} \longrightarrow \mathcal{E}_{\infty} \otimes \mathcal{E}_{\infty}$.

Preuve Considérons le morphisme canonique $\Delta^{c}: \mathcal{C}$ om $\longrightarrow \mathcal{C}$ om $\otimes \mathcal{C}$ om. Il existe une application $\Delta$ telle que le diagramme ci-dessous commute:

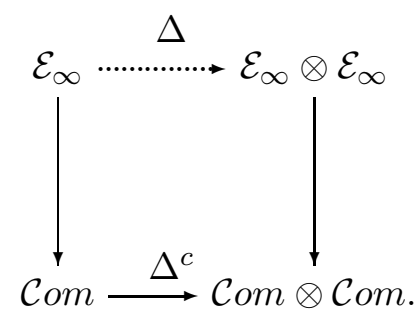

L'existence de $\Delta$ est une conséquence de la propriété de relèvement à gauche par rapport à la fibration triviale $\mathcal{E}_{\infty} \otimes \mathcal{E} \infty \longrightarrow \mathcal{C}$ om $\otimes \mathcal{C}$ om .

Corollaire 4.2 Soient $A$ et $B$ deux $\mathcal{E}_{\infty}$-algèbres alors $A \otimes B$ possède une structure naturelle de $\mathcal{E}_{\infty}$-algèbre.

Preuve L'objet $A \otimes B$ est une $\mathcal{E}_{\infty} \otimes \mathcal{E}_{\infty}$-algèbre. En conséquence, si on a un morphisme $\Delta: \mathcal{E}_{\infty} \longrightarrow \mathcal{E}_{\infty} \otimes \mathcal{E}_{\infty}$, alors on peut utiliser la restriction de structure.

On a des morphismes canoniques de $\mathcal{E}_{\infty}$-algèbres $A \longrightarrow A \otimes B, B \longrightarrow A \otimes B$. De la propriété universelle du coproduit, on obtient un morphisme:

$$
p: A \amalg B \longrightarrow A \otimes B .
$$

On a le résultat suivant:

Proposition 4.6 Soient $A$ et $B$ deux $\mathcal{E}_{\infty}$-algèbres cofibrantes, alors le morphisme ci-dessus:

$$
p: A \amalg B \longrightarrow A \otimes B
$$

est une fibration triviale. 
Preuve Pour commencer, on considère le morphisme de $R$-modules différentiels gradués:

$$
s: A \otimes B \longrightarrow A \amalg B
$$

tel que $s(a \otimes b)=1_{2} \otimes a \otimes b$. V. Hinich a démontré [20] que $s$ est un quasiisomorphisme. On vérifie aisément que: $p(s(a \otimes b))=a \otimes b$. La propriété s'ensuit.

La proposition suivante est une conséquence directe de la proposition 4.5:

Proposition 4.7 Le foncteur $\Omega_{b i}^{\mathcal{E}_{\infty}}$ est un foncteur de la catégorie des ensembles bisimpliciaux dans la catégorie des $\mathcal{E}_{\infty}$-algèbres.

On a également:

Proposition 4.8 Soient $A$ une $\mathcal{E}_{\infty}$-algèbre quasi-libre 1-connexe et $A \longrightarrow$ $A \amalg_{\tau} \mathcal{E}_{\infty}(V)$ un morphisme quasi-libre. Alors on a une suite spectrale de second terme:

$$
E_{2}^{p, q}=\pi^{p}\left(A \otimes \pi^{q}\left(\mathcal{E}_{\infty}(V)\right)\right)
$$

si $\pi^{*}(A)$ ou $\left.\pi^{*}\left(\mathcal{E}_{\infty}(V)\right)\right)$ sont finis (finis en chaque dimension) celle-ci converge vers $\pi^{p+q}\left(A \amalg_{\tau} \mathcal{E}_{\infty}(V)\right)$ dès que $A$ et $\mathcal{E}_{\infty}(V)$ sont connexes.

Preuve On filtre l'algèbre $A$ par le degré différentiel. De cette filtration on déduit une filtration de $A \amalg_{\tau} \mathcal{E}_{\infty}(V)$. En effet, la filtration sur $A$ donne une filtration sur $A \otimes B$, et par suite via le morphisme $p$ une filtration sur $A \amalg \mathcal{E}_{\infty}(V)$, ce qui permet de construire une suite spectrale.

Afin de déterminer le second terme de cette suite spectrale, on se ramène au cas d'un coproduit $A \amalg \mathcal{E}_{\infty}(V)$. En effet, d'après l'hypothèse de 1-connexité de $A$, les suites spectrales associées à $A \amalg_{\tau} \mathcal{E}_{\infty}(V)$ et $A \amalg \mathcal{E}_{\infty}(V)$ ont même second terme.

On remarque que pour un coproduit cette suite spectrale dégénère au terme $E_{2}$. D'après le quasi-isomorphisme $A \amalg \mathcal{E}_{\infty}(V) \longrightarrow A \otimes \mathcal{E}_{\infty}(V)$, on en déduit que:

$$
E_{2}^{p, q}=\pi^{p}\left(A \otimes \pi^{q}\left(\mathcal{E}_{\infty}(V)\right)\right) .
$$

Les algèbres $A$ et $\mathcal{E}_{\infty}(V)$ étant supposées connexes, on obtient une suite spectrale du premier quadrant. Cette suite spectrale converge vers $\pi^{p+q}\left(A \amalg_{\tau}\right.$ $\left.\mathcal{E}_{\infty}(V)\right)$ 
Le modèle de la fibre Soit $p: X \longrightarrow Y$ une fibration de fibre $F$, avec $Y$ supposé 1-connexe, on suppose que la cohomologie de $Y$ ou de $F$ est finie. On se donne $\mathcal{M}_{Y}$ un modèle cofibrant de $\Omega^{\mathcal{E}_{\infty}}(Y)$. On fixe un modèle quasi-libre de

$$
\mathcal{M}_{Y} \stackrel{\phi_{Y}}{\longrightarrow} \Omega^{\mathcal{E} \infty}(Y) \stackrel{p^{*}}{\longrightarrow} \Omega^{\mathcal{E} \infty}(X) .
$$

Soit

$$
p^{\prime}: \mathcal{M}_{Y} \longrightarrow \mathcal{M}_{Y} \amalg_{\tau} \mathcal{M}_{F} .
$$

On va montrer que la $\mathcal{E}_{\infty}$-algèbre $\mathcal{M}_{F}$ est un modèle cofibrant de $F$.

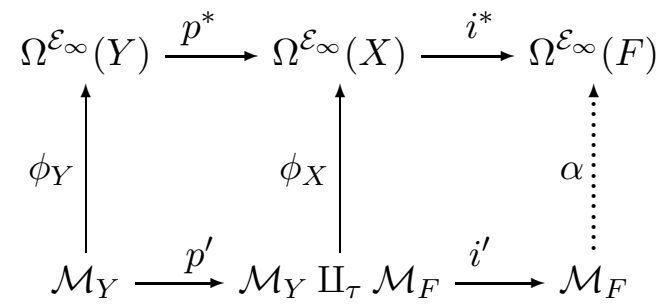

Comme $\mathcal{M}_{F}$ est la cofibre de $p^{\prime}$ il existe un morphisme $\alpha: \mathcal{M}_{F} \longrightarrow \Omega^{\mathcal{E}_{\infty}}(F)$ complétant le diagramme.

Théorème 4.2 Le morphisme $\alpha: \mathcal{M}_{F} \longrightarrow \Omega^{\mathcal{E}_{\infty}}(F)$ est une fibration triviale.

Preuve On a par définition $\alpha i^{\prime}=i^{*} \phi_{X}: \mathcal{M}_{Y} \amalg_{\tau} \mathcal{M}_{F} \longrightarrow \Omega^{\mathcal{E}_{\infty}}(F)$. Or les morphismes $\phi_{X}, i^{*}$ et $i^{\prime}$ sont des fibrations, on en déduit que $\alpha$ est aussi une fibration.

Appliquons le foncteur $\Omega^{\mathcal{E}_{\infty}}$ au diagramme d'ensembles bisimpliciaux:

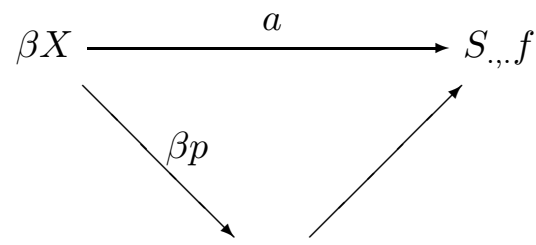

$\beta Y$

On obtient:

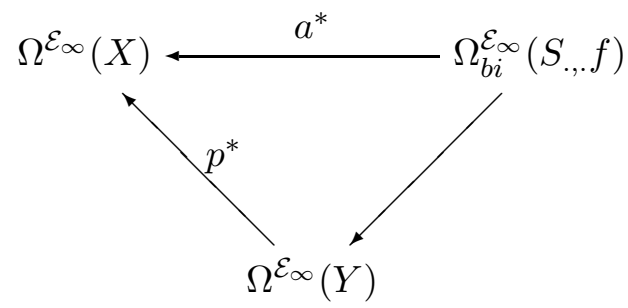

où le morphime $a^{*}$ est une fibration acyclique dans la catégorie des $R$-modules différentiels. Considérons le diagramme suivant: 


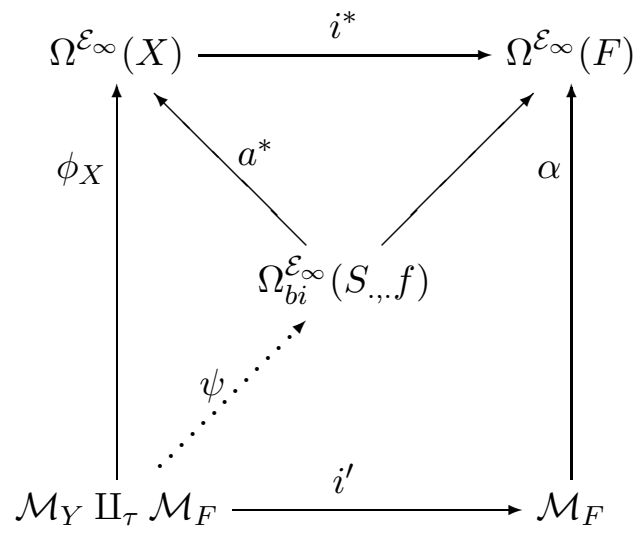

L'existence de $\psi$ (morphisme dans $\mathbf{R} \mathcal{M}_{\mathbf{d g}}$ ) est assurée par le fait que $a^{*}$ est une fibration triviale.

Ce morphisme induit un morphisme de suites spectrales. En particulier, on a:

$$
\psi_{2}^{p, q}: \pi^{p}\left(\mathcal{M}_{Y} \otimes \pi^{q}\left(\mathcal{M}_{F}\right)\right) \longrightarrow H^{p}\left(B ; H^{q}(F ; R)\right)
$$

et

$$
\begin{gathered}
\psi_{2}^{p, 0}=\pi\left(\phi_{Y}\right): \pi^{p}\left(\mathcal{M}_{Y}\right) \longrightarrow H^{p}(B ; R) \\
\psi_{2}^{0, q}=\pi(\alpha): \pi^{q}\left(\mathcal{M}_{F}\right) \longrightarrow H^{q}(F ; R)
\end{gathered}
$$

On sait que $\phi_{X}$ est un quasi-isomorphisme et que $\psi_{2}^{p, 0}=\pi\left(\phi_{Y}\right)$ est un isomorphisme. On en déduit que $\psi_{2}^{0, q}=\pi(\alpha)$ est aussi un isomorphisme par un théorème de comparaison de suites spectrales ([29], section 3.1.1).

\subsection{Transgression dans la suite spectrale de Leray-Serre}

On fixe $R=k$ un corps. Rappelons la définition classique de la transgression [10].

Définition 4.2 Soit $F \stackrel{i}{\longrightarrow} Y \stackrel{p}{\longrightarrow} X$ une fibration on considère le diagramme:

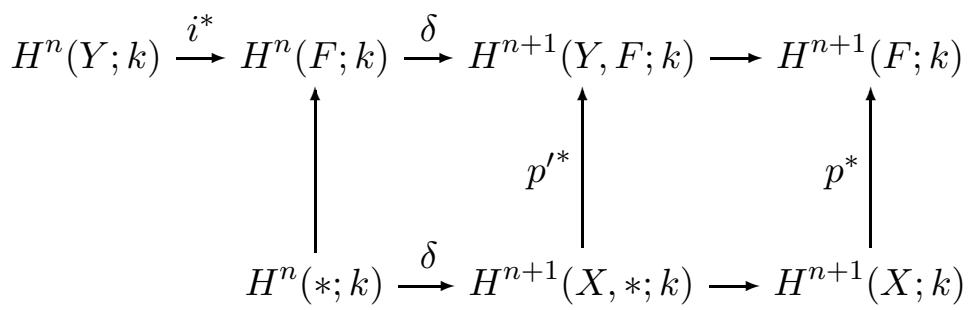

On dit que $[x] \in H^{n+1}(X, * ; k)$ transgresse $[y] \in H^{n}(F ; k)$ si $p^{\prime *}([x])=\delta([y])$. 
On suppose que $\pi_{1}(X)$ opère trivialement sur $H^{n}(F, k)$. On rappelle que, pour la suite spectrale de Leray-Serre associée à cette fibration, on a $E_{n+1}^{0, n} \subset E_{2}^{0, n} \cong$ $H^{n}(F, k)$. Il est équivalent de dire que $[x]$ est une transgression de $[y] \in E_{n+1}^{0, n}$, si $[x]$ se projette sur $d_{n+1}[y]$ via l'application:

$$
H^{n+1}(X, k)=E_{2}^{n+1,0} \longrightarrow E_{n+1}^{n+1,0} .
$$

Proposition 4.9 Soit $[x]$ une transgression de $[y]$; pour tout représentant $y \in Z^{n} \Omega^{\mathcal{E}_{\infty}}(F)$ de $[y]$ et tout représentant $x \in Z^{n+1} \Omega^{\mathcal{E}_{\infty}}(X, *)$ de $[x]$, il existe $u \in \Omega^{\mathcal{E}_{\infty}}(Y)$ tel que $i^{*}(u)=y$ et $d u=q^{*}\left(\alpha_{0}(x)\right)$.

Preuve On considère le diagramme suivant:

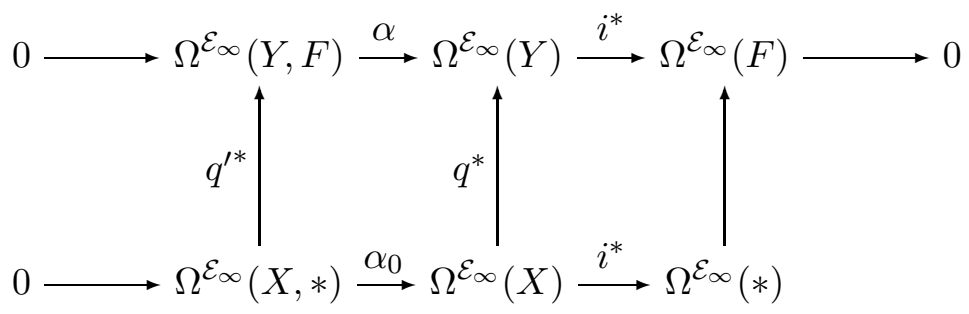

Soit $y \in Z^{n} \Omega^{\mathcal{E}_{\infty}}(F)$. Comme le morphisme $i^{*}$ est surjectif, il existe $u_{1} \in$ $\Omega^{\mathcal{E}_{\infty}^{n}}(Y)$ tel que $i^{*}\left(u_{1}\right)=y$. Par hypothèse, puisque $[x]$ est une transgression de $[y]$, on a $q^{\prime *}([x])=\delta([y])$. Il existe $\lambda \in Z^{n+1} \mathcal{E}_{\infty}(Y, F), \lambda_{1} \in Z^{n} \Omega^{\mathcal{E}_{\infty}}(Y, F)$ tels que $q^{\prime *}(x)=\lambda+d \lambda_{1}$ et $\alpha(\lambda)=d\left(u_{1}\right)$. On en déduit $\alpha\left(q^{\prime *}(x)\right)=\alpha(\lambda)+\alpha\left(d \lambda_{1}\right)$. C'est-à-dire $q^{*}\left(\alpha_{0}(x)\right)=d\left(u_{1}+\alpha\left(\lambda_{1}\right)\right)$. Comme on a aussi $i^{*}\left(u_{1}+\alpha\left(\lambda_{1}\right)\right)=y$, on en conclut que $u=u_{1}+\alpha\left(\lambda_{1}\right)$ convient.

La transgression algébrique On travaille désormais sur $\overline{\mathbb{F}_{p}}$ la clôture algébrique de $\mathbb{F}_{p}$. On suppose que $A \longrightarrow A \amalg_{\tau} \mathcal{O}(V)$ est une extension libre, on note $i: A \longrightarrow A \amalg_{\tau} \mathcal{O}(V)$ et $p: A \amalg_{\tau} \mathcal{O}(V) \longrightarrow \mathcal{O}(V)$ les morphismes canoniques. On suppose aussi que $A$ est 1 -connexe et que $\mathcal{O}(V)$ est connexe. On s'intéresse à la transgression dans la suite spectrale définie dans la proposition 4.8 .

Définition 4.3 Un élément de $[a] \in \pi^{n+1}(A)$ transgresse $[v] \in E_{n+1}^{0, n} \subset E_{2}^{0, n} \cong$ $\pi^{n}(\mathcal{O}(V))$, si $[a]$ se projette sur $d_{n+1}(v) \in E_{n+1}^{n+1,0}$.

Théorème 4.3 (Théorème de Kudo algébrique) Si un élément $[a] \in \pi^{n+1}(A)$ est une transgression de $[v] \in \pi^{n}(\mathcal{O}(V))$, alors $\mathcal{P}^{s}([a])$ transgresse $\mathcal{P}^{s}([v])$ et $\beta \mathcal{P}^{s}([a])$ transgresse $-\beta \mathcal{P}^{s}([v])$. 
Preuve La preuve reprend les arguments développés par J.P. May dans [35] (théorèmes 3.3 et 3.4). On applique le théorème 3.3 de [35] à $i$ et $p$. C'està-dire que l'on construit un morphisme de "suspension" $\sigma: \operatorname{Ker}\left(\pi^{*}(i)\right) \longrightarrow$ $\operatorname{Coker}\left(\pi^{*}(p)\right)$ en posant $\sigma([a])=[p(u)]$ tel que $[a] \in \operatorname{Ker}\left(\pi^{*}(i)\right)$ et $d(u)=i(a)$. Et on montre que ce morphisme de suspension commute aux opérations de Steenrod. On a les formules: $\sigma \mathcal{P}^{s}([a])=\mathcal{P}^{s}(\sigma[a])$ et $\sigma \beta \mathcal{P}^{s}([a])=-\beta \mathcal{P}^{s}(\sigma[a])$. La deuxième partie de la preuve est aussi une adaptation ad hoc de la preuve du théorème 3.4: si $[v] \in \pi^{n}(\mathcal{O}(V))$ est transgressif alors il est représenté par un élément $p(u)$ tel que $d(u)=i(a)$. Le résultat est une conséquence des formules de commutation des opérations de Steenrod à la suspension.

Remarque le théorème de Kudo classique est alors un corollaire du résultat précédent appliqué au modèle de la suite spectrale de Leray-Serre.

\section{Références}

[1] H.J. Baues, M. Jibladze, A. Tonks: Cohomology of monoids in monoidal categories, Operads: Proceedings of Renaissance Conferences (Hartford, CT; Luminy, 1995), 137-165, Contemp. Math., 202, Amer. Math. Soc., Providence, RI, 1997.

[2] C. Berger, B. Fresse: Combinatorial operad actions on cochains, preprint (2001).

[3] J.M. Boardman, R.M. Vogt: Homotopy invariant structures on topological spaces, Lecture Notes in Math., Springer-Verlag.

[4] A.K. Bousfield, V.K.A.M. Gugheneim: On P.L. de Rham theory and rational homotopy type, Memoirs A.M.S, t.8, 179 (1976).

[5] H. Cartan: Théories cohomologiques, Inventiones Mathematicae 3, 172-178 (1967).

[6] A. Dold: Ueber die Steenrodschen Kohomologieoperationen, Ann. of Math (2) 73, 258-294 (1961).

[7] N. Dupont, K. Hess: Noncommutative algebraic models for fiber squares, Math. Ann. 314, no. 3, 449-467 (1999).

[8] A. Dress: Zur Spectralsequenz von Faserungen, Inventiones Mathematicae 35, 261-271 (1976).

[9] W.G. Dwyer, J. Spalinski: Homotopy theory and model categories, Handbook of algebraic topology 73-126 (North-Holland) (1995).

[10] S. Eilenberg, S. Maclane: Relations between homology and homotopy groups, Ann. of Math. 46, 480-509 (1945).

[11] S. Eilenberg, S. Maclane: Acyclic models, Amer. J. of Math. 79, 189-199 (1953). 
[12] Y. Felix, S. Halperin, J.C. Thomas: Differential graded algebras in topology, Handbook of algebraic topology 829-865 (North Holland) (1995).

[13] Y. Felix, S. Halperin, J.C. Thomas: Rational Homotopy Theory, Graduate Texts in Mathematics 205, Springer Verlag (2000).

[14] B. Fresse: Cogroups in algebras over an operad are free algebras, Comment. Math. Helv. 73, 637-676 (1998).

[15] E. Getzler, J.D.S Jones: Operads, homotopy algebra and iterated integrals for double loop spaces, preprint (1994).

[16] V. Ginzburg, M. Kapranov: Koszul duality for operads, Duke J.Math.(1)76, 203-272 (1994).

[17] P.P. Grivel, Formes différentielles et suites spectrales, Ann. de Inst. Fourier (Grenoble) 29, 17-37 (1979).

[18] S. Halperin: Lectures on minimal models, Mémoire de la S.M.F. 9/10 (1983).

[19] V. Hinich: Homological algebra of homotopy algebras, Comm. Algebra 25 no. 10, 3291-3323 (1997).

[20] V. Hinich: Virtual operad algebras and realization of homotopy types, J. Pure Appl. Algebra 159, no. 2-3, 173-185, (2001).

[21] V. Hinich, V. Schechtmann: Homotopy limits of homotopy algebras, in "Ktheory: algebra, geometry, arithmetic", Lecture Notes in Math. 1289, 240-264.

[22] P.S. Hirschorn: Localization of models categories, Preprint (1998).

[23] M. Hovey; Model categories, Mathematical Surveys and Monographs, 63. American Mathematical Society, 1999.

[24] M. Karoubi: Formes différentielles non commutatives et cohomologie à coefficients arbitraires, Transactions of the A.M.S 347, 4277-4299 (1995).

[25] M. Karoubi: Formes différentielles non commutatives et opérations de Steenrod, Topology 34, 699-715 (1995).

[26] I. Kriz, J.P. May: Operads, algebras, modules and motives, Astérisque, 233 (1995).

[27] D. Lehmann: Théorie homotopique des formes différentielles, Astérisque, 45 (1977).

[28] J.L. Loday: La renaissance des opérades, Séminaire Bourbaki 1994-1995, Astérisque, 236 47-74 (1996).

[29] J. McCleary: A user's guide to spectral sequences, Mathematics Lecture series 12, Publish or Perish (1985).

[30] M. Majewski: Rational homotopical models and uniquess, Memoir of the American Mathematical Society, 143 (2000).

[31] M. Mandell: $E_{\infty}$-algebras and p-adic homotopy theory, Topology (1) 40, 43-94 (2001).

[32] M. Mandell: Cochains and homotopy type, preprint (2001). 
[33] M. Markl: Models for operads, Comm.Algebra.(24)4, 1471-1500 (1996).

[34] J.P. May: The geometry of iterated loop spaces, Lecture Notes in Math., Springer-Verlag 271 (1972).

[35] J.P. May: A general algebraic approach to Steenrod operations, Lecture Notes in Math. 168 153-231.

[36] D. Quillen: Homotopical Algebra, Lecture Notes in Math. 43.

[37] N. Spaltenstein: Resolutions of unbounded complexes, Compositio Mathematica 65 (1988) 121-154.

[38] E.H. Spanier: Algebraic Topology, McGraw-Hill series in higher mathematics (1966).

[39] D. Stanley: Determining closed model category structure, Preprint (1998).

[40] J. Smith: Iterating the cobar construction, Mem. Amer. Math. Soc. 109, 524 (1994).

[41] V.A. Smirnov: Homotopy theory of coalgebras, Izv. Akad. Nauk SSSR Ser. Mat. 49, $n^{0} 6,1302-1321$ (1985).

[42] V.A. Smirnov: Lie algebras over operads and their application to homotopy theory, Izv. Math. 62, $n^{0} 3$, 549-580 (1998).

[43] D. Sullivan: Infinitesimal computations in topology, Publ. I.H.E.S. 47 (1977), 269-331.

[44] R. Swan: Thom's theory of differential forms on simplicial sets, Topology 14, 271-273 (1975).

Centre di Recerca Matemática, Institut d'Estudis Catalans

Apartat 50 E - 08193 Bellaterra, Spain

Email: dchataur@crm.es

Received: 17 October 2001

Algebraic 6 Geometric Topology, Volume 2 (2002) 